MATHEMATICS OF COMPUTATION

Volume 76, Number 259, July 2007, Pages 1317-1339

S 0025-5718(07)01971-0

Article electronically published on February 23, 2007

\title{
APPROXIMATION METHODS FOR THE MUSKHELISHVILI EQUATION ON SMOOTH CURVES
}

\author{
V. DIDENKO AND E. VENTURINO
}

\begin{abstract}
We investigate the possibility of applying approximation methods to the famous Muskhelishvili equation on a simple closed smooth curve $\Gamma$. Since the corresponding integral operator is not invertible the initial equation has to be corrected in a special way. It is shown that the spline Galerkin, spline collocation and spline qualocation methods for the corrected equation are stable, and the corresponding approximate solutions converge to an exact solution of the Muskhelishvili equation in appropriate norms. Numerical experiments confirm the effectiveness of the proposed methods.
\end{abstract}

\section{INTRODUCTION}

Let $\Gamma$ be a simple closed Lyapunov curve in the complex plane $\mathbf{C}$ and let $\gamma$ be a 1 -periodic parametrization of $\Gamma$, which maps the interval $[0,1) 1-1$ and onto $\Gamma$ and $\gamma^{\prime}(s) \neq 0$ for every $s \in[0,1]$. This curve divides the complex plane into two domains. The interior domain will be denoted by $D$, and throughout this paper we always assume that $0 \in D$.

Let $H^{\sigma}, \sigma \in \mathbf{R}$, denote the periodic Sobolev space of order $\sigma$, i.e. the completion of the set $C^{\infty}$ of all 1 -periodic infinitely differentiable functions on $\mathbf{R}$ with respect to the norm

$$
\|\phi\|_{\sigma}=\left[\left|\hat{\phi}_{0}\right|^{2}+\sum_{j \in \mathbf{Z}, j \neq 0}\left|\hat{\phi}_{j}\right|^{2}|2 \pi j|^{2 \sigma}\right]^{\frac{1}{2}}
$$

where $\hat{\phi}_{j}, j \in \mathbf{Z}$, stands for the Fourier coefficients of the function $\phi$. If a function $\psi(t)$ is defined on the countour $\Gamma$, it can be identified with a 1 -periodic function on $\mathbf{R}$ by setting

$$
\psi(s)=(\psi \circ \gamma)(s)=\psi(\gamma(s)), \quad s \in \mathbf{R} .
$$

Now, by $H^{\sigma}(\Gamma)$ we denote the closure of $C^{\infty}(\Gamma)$ with respect to the norm $\|\psi \circ \gamma\|_{\sigma}$. Note that in the sequel we often use notations $L_{2}(\Gamma)$ and $W_{2}^{1}(\Gamma)$ for the spaces $H^{0}(\Gamma)$ and $H^{1}(\Gamma)$ respectively. Moreover all spaces of this paper are considered over the field of the real numbers $\mathbf{R}$, so all the operators under consideration are linear. Some of these operators are however not linear if considered as operators on spaces over the field of complex numbers.

Received by the editor January 26, 2006 and, in revised form, June 20, 2006.

2000 Mathematics Subject Classification. Primary 65R20.

The first author thanks INDAM for the support provided to him during his June 2002 visit to the University of Torino, where most of this research was carried out. He was also partially supported by UBD via Grant UBD/PNC2/2/RG/1(49).

(C) 2007 American Mathematical Society Reverts to public domain 28 years from publication 1317 
Let $f \in W_{2}^{1}(\Gamma)$. We consider the Muskhelishvili equation on $\Gamma,[19$, §18] and [16, pp. $42-43]$, viz.

(3) $R x(t) \equiv-\overline{x(t)}-\frac{1}{2 \pi i} \int_{\Gamma} \overline{x(\tau)} d \log \frac{\bar{\tau}-\bar{t}}{\tau-t}-\frac{1}{2 \pi i} \int_{\Gamma} x(\tau) d \frac{\bar{\tau}-\bar{t}}{\tau-t}=f_{0}(t), \quad t \in \Gamma$,

where the bar denotes complex conjugation and

$$
f_{0}(t)=-\frac{1}{2} \overline{f(t)}+\frac{1}{2 \pi i} \int_{\Gamma} \frac{\overline{f(\tau)}}{\tau-t} d \tau .
$$

The operator $R$, and then also $R_{1}$ defined below after (7), maps $L_{2}(\Gamma)$ into itself; see [12, 13]. Note that if $f \in L_{p}, 1<p<\infty$, then $f_{0} \in L_{p}$ as well [14, p. $18-$ 24]. It is well known, [16, pp. $42-43$ ], 17], 19, Chapter 4], that equation (3) is closely connected to different problems of elasticity theory and the theory of slow viscous flows. In fact, a number of boundary value problems for the biharmonic operator in the domain $D$ can be reduced to equation (3). This makes equation (31) a very valuable object for application of different numerical procedures because it allows us to essentially reduce computational costs while considering the corresponding problems for partial differential equations. Nevertheless, up to now the Muskhelishvili equation received only scant attention in numerical analysis. The reason for this might lie in the fact that the operator $R$ is invertible neither on the space $W_{2}^{1}(\Gamma)$ nor on $L_{p}(\Gamma)$; see e.g. [11, [12, [19]. In particular, equation (3) is solvable if and only if

$$
\operatorname{Re} \int_{\Gamma} \overline{f(t)} d t=0
$$

Therefore, no popular projection method such as Galerkin, collocation, qualocation and so on can be applied to equation (3) directly.

Let us mention here that the Muskhelishvili equation was conceived as a tool for studying the following boundary value problem, see [19, Chapter 4],

$$
\psi(t)+\overline{\phi(t)}+\bar{t} \phi^{\prime}(t)=\overline{f(t)}, \quad t \in \Gamma,
$$

for two analytic functions $\phi$ and $\psi$ in $D$. Therefore most of the known numerical methods for (3) are concerned with the approximate solution of the boundary value problem (6), 1], 2], 17]. In the case where $\Gamma$ is the unit circle, N.I. Muskhelishvili proposed to use Fourier series expansion for solving the problem (3). However, if $\Gamma$ differs from the unit circle, such a method cannot be applied, thus conformal mappings should first be used, 1, 2]. Simultaneously, it was observed that this approach gives good results for the contours that, in a sense, are close to the unit circle. However, even for ellipses with a small eccentricity such a method can encounter certain difficulties. Moreover, it is worth mentioning that the operator corresponding to the left hand side of (6) is not invertible either. Hence projection methods for (6) are not going to be stable. That is why the authors of the papers mentioned tried to modify the initial spaces to obtain invertibility of corresponding operators. Usually such procedures require a lot of effort, and it is not always completely clear how to properly construct appropriate spaces.

In the present paper we use different ideas. Instead of modifying the initial spaces we prefer to correct the operator $R$ of (3), in a way such that the new operator $R_{1}$ is already invertible on $L_{2}(\Gamma)$ and, what is equally important, a new equation can be used for finding approximate solutions of (3). Such an approach was probably first mentioned by P.I. Perlin and Yu.N. Shalyukhin in [20, 21]. However, in [20] only 
a table with 7 exact and approximate values for the solution of the Muskhelishvili equation on the unit circle is provided, without any description of the numerical method used. In [21] the method was described in more detail for a special contour but again no proof of its stability was given.

The theoretical tool for our analysis which opens a way for finding the approximate solution of the Muskhelishvili equation is provided by the following result 8 .

Proposition 1. Let $\Gamma$ be a simple closed Lyapunov curve and let the operator $T: L_{2}(\Gamma) \longrightarrow L_{2}(\Gamma)$ be defined by

$$
T x(t) \equiv \frac{1}{2 \pi i} \int_{\Gamma} \frac{x(\tau) d \tau}{\tau}+\frac{1}{t} \frac{1}{2 \pi i} \int_{\Gamma}\left(\frac{x(\tau)}{\tau^{2}} d \tau+\frac{\overline{x(\tau)}}{\bar{\tau}^{2}} d \bar{\tau}\right) .
$$

Then the operator $R_{1}=R+T$ is invertible on $L_{2}(\Gamma)$. If , in addition, $f \in W_{2}^{1}(\Gamma)$ and satisfies condition (5), then the solution of the equation

$$
R_{1} x=f_{0}
$$

belongs to the space $W_{2}^{1}(\Gamma)$ and is simultaneously a solution of the Muskhelishvili equation (3).

In [8] a spline Galerkin and a quadrature method was studied in the case where $\Gamma$ was assumed to be a piecewise smooth curve. The stability of the approximation methods was shown to depend on the invertibility of some quite complicated operators from a Toeplitz algebra.

In the present paper the curve $\Gamma$ is assumed to be smooth. This leads to a very nice result. Namely, all approximation methods under consideration converge without any additional conditions. It is also worth mentioning that in 8 the authors used only piecewise constant splines. However, it would be desirable to use splines of higher degree since such splines could be more effective in the calculation of the corresponding integrals. In contrast to 8 then, we consider here these higher order splines and we also study the collocation and qualocation methods. Note that for splines of higher degree the applicability of the corresponding Galerkin method for piece-wise contours was first established in [9]. The authors of [9] also mentioned that for smooth boundaries their result can be simplified. However, in the present paper we give a stability proof which is well adjusted to the case of smooth boundaries and does not follow from that in 8 . Finally, note that condition (5) is assumed to hold throughout this paper.

For the analysis of other boundary element methods for biharmonic Dirichlet problem in smooth domains we refer the reader to 4, 5. Let us only note that for some boundaries, called critical, the integral operators considered in [4, 5] are not invertible. Therefore, the methods like Galerkin and collocation are not directly applicable. Although such situations are relatively rare, it is not a priori clear whether those integral operators are invertible or not. Their invertibility depends on the logarithmic capacity of the curve, and for curves of any shape, including circles, there are cases where the corresponding integral operators on such curves are not invertible [6]. On the other hand, the approach presented here is free from such kinds of problems, i.e. these methods converge for any smooth contour.

Note that although this paper deals only with spline approximation methods, the use of the corrected operator $R_{1}$ opens the way for the application of projection methods based on other approximating aggregates. In this way for instance the 
main trigonometric methods, see [15], 22], could also be applied to equation (8). In some cases these can improve the order of convergence, but the final results will depend heavily on the smoothness of the contour $\Gamma$.

\section{Approximate solutions}

Let us recall some definitions and standard notations. For any operator $B$ defined on a Banach space $X$, the range of $B$ on $X$ is denoted by $\operatorname{im}_{X} B$ or simply by $\operatorname{im} B$. Now consider a sequence of subspaces $X_{1} \subset X_{2} \subset \ldots \subset X_{n} \subset \ldots$ in the Banach space $X$ and a sequence of projections $Q_{n}, n \in \mathbf{N}$, with the property $\operatorname{im} Q_{n}=X_{n}$ for each $n=1,2, \ldots$. If $A$ is a linear operator on $X$ and if $\left\{A_{n}\right\}$ is a sequence of linear operators on the spaces $X_{n}$ such that $A_{n} Q_{n}$ converges to $A$ strongly as $n \longrightarrow \infty$, then for the approximate solution of the equation

$$
A x=y, \quad x, y \in X,
$$

one can use the sequence of equations

$$
A_{n} x_{n}=Q_{n} y, \quad x_{n} \in X_{n}, \quad n \in \mathbf{N} .
$$

Definition 2. A sequence $\left\{A_{n}\right\}$ is said to be stable if there exists $n_{0} \in \mathbf{N}$ such that for all $n \geq n_{0}$ the operators $A_{n}: X_{n} \longrightarrow X_{n}$ are invertible and $\sup _{n \geq n_{0}}\left\|A_{n}^{-1} Q_{n}\right\|<$ $\infty$.

In the sequel we need the following result, which will be used by setting $X \equiv$ $L_{2}(\Gamma)$ when considering the Galerkin method, while we take $X \equiv C(\Gamma)$ for the collocation and qualocation methods.

Theorem 3 ([22, pp. 26-27]). Let $\left\{A_{n}\right\}$ be stable and let $x_{n}, n \geq n_{0}$, be the solution of equation (10). Then

$$
\left\|x-x_{n}\right\| \leq \inf _{v \in X_{n}}\left[\|x-v\|+\left\|A_{n}^{-1}\right\|\left(\left\|A_{n} v-A x\right\|+\left\|y-Q_{n} y\right\|\right)\right] .
$$

Remark. Notice that for the projection method $\left(A_{n}\right), A_{n}=Q_{n} A Q_{n}$, this theorem shows that the error is proportional to $\left\|Q_{n}\right\| E_{n}(x)$ where $Q_{n}, n \in \mathbf{N}$, are the corresponding projection operators and $E_{n}(x)$ denotes the best approximation to the solution by elements from $X_{n}$. The latter allows us to characterize the convergence in terms of the smoothness of the solution $x$ upon showing stability, using Jackson type approximation theorems in suitable subspaces.

In the present paper we consider the stability of approximation methods based on splines. Thus let us describe the spline spaces and the spline projections we use. Let $(f * g)$ denote the convolution of the functions $f$ and $g$,

$$
(f * g)(s)=\int_{\mathbf{R}} f(s-x) g(x) d x .
$$

Consider the characteristic function $\chi=\chi(s), s \in \mathbf{R}$, of the interval $[0,1)$, i.e.

$$
\chi(s)=\left\{\begin{array}{cc}
1 & s \in[0,1) \\
0 & \text { otherwise. }
\end{array}\right.
$$

For any $d \in \mathbf{N}$ introduce the function $\phi^{d}$ by

$$
\phi^{d}(s)=\left(\phi^{0} * \phi^{d-1}\right)(s)
$$

where $\phi^{0}(s)=\chi(s), s \in \mathbf{R}$. It is well known, see e.g. [23], p. 134-139, that the functions $\phi^{d}$ generate spline spaces on $\mathbf{R}$. To be more precise, we fix $d \in \mathbf{N}$ and set

$$
\tilde{\phi}(s)=\phi^{d}(s), \quad s \in \mathbf{R} .
$$


Also, fix a number $n \in \mathbf{N}$ and for each $j \in \mathbf{Z}$ define the function $\tilde{\phi}_{j n}=\tilde{\phi}_{j n}(s)$ by

$$
\tilde{\phi}_{j n}(s)=\tilde{\phi}(n s-j), \quad s \in \mathbf{R} .
$$

Then the set of all linear combinations of $\tilde{\phi}_{j n}, j \in \mathbf{Z}$, is a spline space on $\mathbf{R}$.

Using the above construction one can introduce the corresponding spline spaces on $\Gamma$. Thus, if $\gamma$ is a 1 -periodic parametrization of the curve $\Gamma$, then for any $t \in \Gamma$ we set

$$
\phi_{j n}(t)=\tilde{\phi}_{j n}(s), \quad t=\gamma(s), \quad s \in \mathbf{R} .
$$

Let $S_{n}^{d}=S_{n}^{d}(\Gamma)$ denote the corresponding spline space on $\Gamma$.

2.1. The Galerkin method. An approximate solution $x_{n}=x_{n}(t) \in S_{n}^{d}$ of equation (3) is sought in the form

$$
x_{n}(t)=\sum_{j=0}^{n-1} c_{j} \phi_{j n}(t), \quad t \in \Gamma .
$$

By $(\cdot, \cdot)$ we denote the usual inner product on $\Gamma$, i.e.

$$
(f, g)=\int_{\Gamma} f(t) \overline{g(t)}|d t|, \quad f, g \in L_{2}(\Gamma) .
$$

Note that the norm on $L_{2}(\Gamma)$ generated by this scalar product is equivalent to the one defined by (11), (2).

The unknown coefficients $c_{j}, j=0,1, \ldots, n-1$, of the approximate solution (12) are defined by the following system of algebraic equations:

$$
\left(R_{1} x_{n}, \phi_{k n}\right)=\left(f_{0}, \phi_{k n}\right), \quad k=0,1, \ldots, n-1,
$$

where $R_{1}$ is defined in Proposition 1 .

Theorem 4. Let $\Gamma$ be a simple closed Lyapunov curve, and let $f \in W_{2}^{1}(\Gamma)$. Then there exists $n_{0}$ such that for all $n \geq n_{0}$ the systems of algebraic equations (13) are solvable and the sequence (12) of approximate solutions of equation (8) converges to the exact solution of (3) in the norm of $L_{2}(\Gamma)$.

Proof. Denote by $P_{n}$ the orthogonal projections onto the spline subspaces $S_{n}^{d}(\Gamma)$. Then the systems of algebraic equations (13) are equivalent to the operator equations

$$
P_{n} R_{1} P_{n} x_{n}=P_{n} f_{0}, \quad n=1,2 \ldots
$$

Therefore if we are able to prove the stability of the sequence $\left\{P_{n} R_{1} P_{n}\right\}$, our claim will follow from Proposition 1, Theorem 3 and from the corresponding results of approximation theory. Let $M$ refer to the operator of complex conjugation and let $K$ be the operator defined by

$$
\begin{aligned}
K x(t)=- & \frac{1}{2 \pi i} \int_{\Gamma} \overline{x(\tau)} d \log \frac{\bar{\tau}-\bar{t}}{\tau-t}-\frac{1}{2 \pi i} \int_{\Gamma} x(\tau) d \frac{\bar{\tau}-\bar{t}}{\tau-t} \\
& +\frac{1}{2 \pi i} \int_{\Gamma} \frac{x(\tau) d \tau}{\tau}+\frac{1}{t} \frac{1}{2 \pi i} \int_{\Gamma}\left(\frac{x(\tau) d \tau}{\tau^{2}}+\frac{\overline{x(\tau)}}{\bar{\tau}^{2}} d \bar{\tau}\right)
\end{aligned}
$$

Then the operator $P_{n} R_{1} P_{n}$ can be written as $P_{n} R_{1} P_{n}=-P_{n} M P_{n}+P_{n} K P_{n}$. Using the easily verified equality $M P_{n}=P_{n} M$, we immediately obtain

$$
P_{n} R_{1} P_{n}\left(-P_{n} M P_{n}\right)=P_{n}-P_{n} K M P_{n} .
$$


By Theorem 1 the operator $R_{1}$ is invertible on the space $L_{2}(\Gamma)$. We can thus introduce operators $B_{n}: \operatorname{im} P_{n} \longrightarrow \operatorname{im} P_{n}$ by

$$
B_{n}=-P_{n} M P_{n}+P_{n} R_{1}^{-1} K M P_{n} \text {. }
$$

Then by (16)

$$
\begin{aligned}
\left(P_{n} R_{1} P_{n}\right) B_{n} & =\left(P_{n} R_{1} P_{n}\right)\left(-P_{n} M P_{n}\right)+\left(P_{n} R_{1} P_{n}\right)\left(P_{n} R_{1}^{-1} K M P_{n}\right) \\
& =P_{n}-P_{n} K M P_{n}+P_{n} R_{1} P_{n} R_{1}^{-1} K M P_{n} \\
& =P_{n}-P_{n} R_{1}\left(I-P_{n}\right)\left(R_{1}^{-1} K M\right) P_{n} .
\end{aligned}
$$

But by Lemma 5.21 of 22 the projections $P_{n}$ converge strongly to the identity operator. Since $\Gamma$ is a Lyapunov curve the operator $K$ is compact on $L_{2}(\Gamma)$, 12, [18. Therefore, the sequence $\left\{P_{n} R_{1}\left(I-P_{n}\right) R_{1}^{-1} K M P_{n}\right\}$ converges uniformly to 0 as $n \longrightarrow \infty$. Hence, the operators in the right hand side of (17) are invertible on im $P_{n}$ for all $n$ sufficiently large and the norms of their inverses are bounded, for example by 2 .

This implies the invertibility from the right of the operators $\left(P_{n} R_{1} P_{n}\right)$ and the inequality

$$
\left\|\left(P_{n} R_{1} P_{n}\right)^{-1} P_{n}\right\| \leq 2\left\|B_{n}\right\| \leq 2+2\left\|R_{1}^{-1} K\right\|
$$

for the norms of the right inverses. The left invertibility of $P_{n} R_{1} P_{n}$ can be proved analogously. Thus, the sequence $\left\{P_{n} R_{1} P_{n}\right\}$ is stable, and the first assertion of Theorem 4 follows from Theorems 1 and 3 .

Taking into account the stability of the method, Proposition 1, and inequality (11) one can get error estimates. More precisely, if the right hand side $f$ in (7) belongs to $W_{2}^{1}(\Gamma)$, then $f_{0} \in W_{2}^{1}(\Gamma)$, 14 and the solution $x$ of (8) belongs to $W_{2}^{1}(\Gamma)$ as well, [12]. Therefore, by [22, p. 44]

$$
\left\|x-P_{n} x\right\|_{L_{2}(\Gamma)} \leq \frac{c_{1}}{n}\|x\|_{W_{2}^{1}(\Gamma)}
$$

and

$$
\left\|f_{0}-P_{n} f_{0}\right\|_{L_{2}(\Gamma)} \leq \frac{c_{2}}{n}\left\|f_{0}\right\|_{W_{2}^{1}(\Gamma)}
$$

where $c_{1}, c_{2}$ are constants independent of $x, f_{0}$ and $n$. Hence, using (11) one obtains

$$
\begin{aligned}
\left\|x-x_{n}\right\|_{L_{2}(\Gamma) \leq} \leq & \left\|x-P_{n} x\right\|_{L_{2}(\Gamma)}+\left\|A_{n}^{-1} P_{n}\right\|\left(\left\|P_{n} A P_{n} x-A P_{n} x\right\|_{L_{2}(\Gamma)}\right. \\
& \left.+\left\|A P_{n} x-A x\right\|_{L_{2}(\Gamma)}+\left\|f_{0}-P_{n} f_{0}\right\|_{L_{2}(\Gamma)}\right) \\
\leq & \frac{c_{3}}{n},
\end{aligned}
$$

where the constant $c_{3}$ is independent of $n$. This completes the proof.

2.2. The collocation method. Let $0 \leq \epsilon<1$ be a real number and let $t_{j}^{(n)} \in \Gamma$ be defined as follows:

$$
t_{j}^{(n)}=\gamma\left(\frac{j+\epsilon}{n}\right), \quad j=0,1, \ldots, n-1 .
$$

The approximate solution of equation (3) is again sought in the form (12), but the unknown coefficients $c_{j}, j=0,1, \ldots, n-1$, will be obtained from the system

$$
R_{1} x_{n}\left(t_{j}^{(n)}\right)=f_{0}\left(t_{j}^{(n)}\right), \quad j=0,1, \ldots, n-1 .
$$

The collocation method is considered in the context of the space $C(\Gamma)$, so $X$ in Theorem 3 is identified now with this space. It follows from [22, p. 64], that if $d$ is 
odd and $\epsilon \neq \frac{1}{2}$ or if $d$ is even and $\epsilon \neq 0$, then there exists a uniquely determined interpolation projection $L_{n}$ onto the space $S_{n}^{d}(\Gamma)$ such that

$$
L_{n} f\left(t_{j}^{(n)}\right)=f\left(t_{j}^{(n)}\right) .
$$

Using this notation and recalling the remark after (14), we see that the system (18) is equivalent to the operator equation

$$
L_{n} R_{1} P_{n} x_{n}=L_{n} f_{0}, \quad n \in \mathbf{N} .
$$

However, the projections $L_{n}$ are not defined on the space $L_{2}(\Gamma)$. Hence, to be able to study the collocation method we have to consider the operator $R_{1}$ on a more appropriate space.

Theorem 5. Let $\Gamma$ be a simple closed curve and let its parametrization $\gamma$ be twice continuously differentiable on $[0,1]$. Assume that $f \in W_{2}^{1}(\Gamma)$. Then there exists an integer $n_{0}$ such that equations (20) are solvable for all $n \geq n_{0}$, and the sequence $\left\{x_{n}\right\}_{n \geq n_{0}}$ converges to a solution of equation (3) in the norm of $C(\Gamma)$.

Proof. We now consider the operator $R_{1}$ on the space $C(\Gamma)$. First of all we mention that if $\gamma$ is twice continuously differentiable, then the operator $K$ of (15) is compact on $C(\Gamma)$. Really, this claim is obvious for the operator $T$ because the kernels of the corresponding integral operators are continuous. Therefore consider for instance the operator $T_{1}$,

$$
T_{1} x(t)=\frac{1}{2 \pi i} \int_{\Gamma} x(\tau) d \frac{\bar{\tau}-\bar{t}}{\tau-t}=\int_{\Gamma} K_{1}(t, \tau) x(\tau) d \tau .
$$

The kernel $K_{1}(t, \tau)$ of this integral operator has the form

$$
K_{1}(t, \tau)=\frac{1}{2 \pi i}\left[\frac{(\tau-t) \frac{d \bar{\tau}}{d \tau}-(\bar{\tau}-\bar{t})}{(\tau-t)^{2}}\right] .
$$

Since $\Gamma$ does not have any intersections with itself, the function $K_{1}$ is obviously continuous for $\tau \neq t$. Let us study the behavior of the expression

$$
\Phi_{1}(t, \tau)=\frac{(\tau-t) d \bar{\tau}-(\bar{\tau}-\bar{t}) d \tau}{(\tau-t)^{2}}
$$

when $\tau$ tends to $t$. Setting $\tau=\gamma(\sigma), t=\gamma(s), \sigma, s \in[0,1), \sigma \neq s$ and using the twice continuous differentiability of the function $\gamma$, we get as $\sigma \longrightarrow s$

$$
\Phi_{1}(t, \tau)=\frac{i \operatorname{Im}\left(\overline{\gamma^{\prime \prime}(\sigma)} \gamma^{\prime}(\sigma)\right)+o(1)}{\left[\gamma^{\prime}(\sigma)\right]^{2}}
$$

Hence

$$
\lim _{\tau \rightarrow t} \Phi_{1}(t, \tau)=i \frac{\operatorname{Im}\left(\overline{\gamma^{\prime \prime}(s)} \gamma^{\prime}(s)\right)}{\left[\gamma^{\prime}(s)\right]^{2}},
$$

thus the function $\Phi_{1}(t, \tau)$ is continuous for all $t, \tau$ on $\Gamma$, and the operator $T_{1}$ : $C(\Gamma) \longrightarrow C(\Gamma)$ is compact. The compactness of the remaining integral operator $T_{2}$,

$$
T_{2} x(t)=-\frac{1}{2 \pi i} \int_{\Gamma} \overline{x(\tau)} d \log \frac{\bar{\tau}-\bar{t}}{\tau-t}=\int_{\Gamma} K_{2}(t, \tau) \overline{x(\tau)} d \tau
$$


can be shown in the same way, thus obtaining

$$
\lim _{\tau \longrightarrow t} \Phi_{2}(t, \tau)=i \frac{\operatorname{Im}\left(\overline{\gamma^{\prime \prime}(s)} \gamma^{\prime}(s)\right)}{\left|\gamma^{\prime}(s)\right|^{2}} .
$$

We can now show the invertibility of the operator $R_{1}$ on $C(\Gamma)$. Since $K$ is compact, the standard Fredholm theory implies that the index of the operator $R_{1}$ considered on $C(\Gamma)$ is equal to zero. The space $C(\Gamma)$ is dense in $L_{2}(\Gamma)$ and the index of $R_{1}$ on $L_{2}(\Gamma)$ is equal to zero [8]. Therefore, by [13] the dimensions of the kernels of $R_{1}$ on $L_{2}(\Gamma)$ and on $C(\Gamma)$ coincide. However the operator $R_{1}: L_{2}(\Gamma) \longrightarrow$ $L_{2}(\Gamma)$ is invertible, hence dim ker $\left.R_{1}\right|_{L_{2}(\Gamma)}=0$. This implies dim ker $\left.R_{1}\right|_{C(\Gamma)}=0$. Taking into account that the index of the operator $R_{1}: C(\Gamma) \longrightarrow C(\Gamma)$ is also zero, one obtains the invertibility of the operator $R_{1}$ on the Banach space $C(\Gamma)$.

The next steps mainly follow the Proof of Theorem 4. Representing the operator $L_{n} R_{1} P_{n}$ in the following form:

$$
L_{n} R_{1} P_{n}=-L_{n} M P_{n}+L_{n} K P_{n}
$$

and multiplying the latter expression by the operator

$$
\tilde{B}_{n}=-L_{n} M P_{n}+L_{n} R_{n}^{-1} K M P_{n}
$$

one obtains

$$
\left(L_{n} R_{1} P_{n}\right) \tilde{B}_{n}=P_{n}-L_{n} R_{1}\left(I-L_{n}\right) R_{1}^{-1} K M P_{n} .
$$

Since by Lemma 5.28 of 22 the sequence $\left\{I-L_{n}\right\}_{n \in \mathbf{N}}$ is uniformly bounded, the approximation properties of the splines guarantee that the sequence converges strongly to zero on $C(\Gamma)$. Also observing that the operator $R^{-1} K M$ is compact, we get that for all $n$ large enough the operators $L_{n} R_{1} P_{n}$ : im $P_{n} \longrightarrow \operatorname{im} P_{n}$ are right invertible and their right inverses are uniformly bounded. Since these operators are finite dimensional their invertibility on $\operatorname{im} P_{n}$ follows. Having proved the stability of the sequence $\left\{L_{n} R_{1} P_{n}\right\}$ we again can use Theorem 3 to establish the convergence of the approximate solutions.

2.3. The qualocation method. This method represents a discrete version of the Galerkin method. The qualocation method was routinely used while applying approximate procedures to operator equations, but its systematic study was started by I.H. Sloan in 1988, 24. At present there is a large number of papers devoted to different aspects of the method. Here we consider the stability of the following version of the qualocation method for the Muskhelishvili equation.

Let $0<\epsilon_{1}<\epsilon_{2} \ldots<\epsilon_{m}<1$ and $\omega_{1}, \omega_{2}, \ldots, \omega_{m}$, be positive numbers such that $\omega_{1}+\omega_{2}+\ldots+\omega_{m}=1$ and let $t_{j, \epsilon_{k}}^{(n)}=\gamma\left(\frac{j+\epsilon_{k}}{n}\right), j \in \mathbf{Z}, k=1,2, \ldots, m$. By $Q_{n}$ we denote the quadrature formula

$$
Q_{n}(g)=\sum_{j=0}^{n-1} \sum_{r=1}^{m} \omega_{r} g\left(t_{j, \epsilon_{r}}^{(n)}\right) .
$$

The approximate solution of equation (3) is sought in the form (12) but in contrast to (13) the coefficients $c_{j}, j=0,1, \ldots, n-1$, are determined from the following system of algebraic equations:

$$
Q_{n}\left(R_{1} x_{n}, v\right)=Q_{n}\left(f_{0}, v\right), \quad x_{n} \in S_{n}^{d}(\Gamma),
$$

for all $v \in S_{n}^{0}(\Gamma)$, where $S_{n}^{0}(\Gamma)$ denotes the space of piecewise constant splines on $\Gamma$. Note that such a spline qualocation method, though with the more general 
spline space $S_{n}^{\mu}(\Gamma)$ in place of $S_{n}^{0}(\Gamma)$, was studied in [10] for the Cauchy singular integral equations on the unit circle $\Gamma$.

Theorem 6. Let $\Gamma$ be a simple closed curve such that its parametrization $\gamma$ is a twice continuously differentiable function on $[0,1]$. Assume also that $f \in W_{2}^{1}(\Gamma)$, and let $\epsilon_{r} \in(0,1)$ be real numbers such that $\epsilon_{r} \neq \frac{1}{2}, r=1,2, \ldots, m$, if $d$ is odd. Then there exists an integer $n_{0}$ such that for all $n \geq n_{0}$ equations (21) are solvable and the corresponding approximate solutions $x_{n}$ converge to an exact solution of equation (3) in the norm of $C(\Gamma)$.

Proof. The operators $A_{n}: S_{n}^{d}(\Gamma) \longrightarrow S_{n}^{d}(\Gamma)$ corresponding to the left hand side of (21) can be represented in the form

$$
A_{n}=\sum_{r=1}^{m} \omega_{r} L_{n}^{\epsilon_{r}} R_{1} P_{n}
$$

where $L_{n}^{\epsilon_{r}}$ denotes the interpolation projection onto the spline space $S_{n}^{d}(\Gamma)$ satisfying the property

$$
L_{n}^{\epsilon_{r}} u\left(t_{j, \epsilon_{r}}\right)=u\left(t_{j, \epsilon_{r}}\right), \quad j=0,1, \ldots, n-1 .
$$

Since $R_{1}=-M+K$ and $L_{n}^{\epsilon_{r}} M P_{n}=M P_{n}=L_{n}^{\epsilon_{1}} M P_{n}$ for any $r=1,2, \ldots, m$ we can rewrite (22) as

$$
A_{n}=-L_{n}^{\epsilon_{1}} M P_{n}+L_{n}^{\epsilon_{1}} K P_{n}+\sum_{r=2}^{m} \omega_{r}\left(L_{n}^{\epsilon_{r}}-L_{n}^{\epsilon_{1}}\right) K P_{n} .
$$

The sequence $\left\{L_{n}^{\epsilon_{r}}-L_{n}^{\epsilon_{1}}\right\}$ converges strongly to 0 on the space $C(\Gamma)$ as $n \longrightarrow \infty$. Taking into account the compactness of $K$ we deduce that

$$
\left\|\sum_{r=2}^{m} \omega_{r}\left(L_{n}^{\epsilon_{r}}-L_{n}^{\epsilon_{1}}\right) K P_{n}\right\| \longrightarrow 0
$$

as $n \longrightarrow \infty$. Now we can proceed as in the proofs of Theorems 4 and 5 .

\section{Discussion}

We implemented both the Galerkin and collocation methods on several examples, using splines of order $m=d+1$. We performed an extensive investigation on the performance of the proposed schemes, examining in particular the behavior of the code as a function of the various available parameters.

Note that the major computational effort lies in solving systems of algebraic equations and thus it is the same for each example and the same approximation method if the matrices $M$ have the same size. The estimates on the conditioning of the system are obtained from the standard Matlab function $\operatorname{cond}(M, p)$ with $p=2$ and $p=\infty$. Note that the conditioning is the same for all the examples, as it is related with their left hand sides, which all contain the same operator. The examples differ indeed only in their right hand sides.

Table 1 contains the condition numbers of the collocation scheme in the $L_{\infty}$ norm, when $\Gamma$ is the unit circle, for various choices of the parameter $\epsilon$ and order of the splines. The number of grid points is even. The case $\epsilon=.5$ that is not entirely covered by Theorem 5 , is taken here on purpose. It gives ill-conditioning if the order $m$ of splines is even, while the scheme appears to be better behaved the closer to the endpoints of $[0,1]$ the choice of $\epsilon$ is. For $m$ odd, the reverse occurs; 
TABLE 1. Conditioning with collocation scheme - even number of nodes

\begin{tabular}{cccccc}
\hline \hline \multicolumn{5}{c}{$\|M\|_{\infty}\left\|M^{-1}\right\|_{\infty}$} \\
\hline$\epsilon=.01$ & $n$ & $m=2$ & $m=3$ & $m=4$ & $m=5$ \\
& 16 & 33.843 & 677.30 & 55.489 & 2002.5 \\
& 32 & 35.949 & 658.84 & 57.022 & 1922.1 \\
& 64 & 36.925 & 653.40 & 59.140 & 1905.5 \\
& 128 & 37.418 & 650.80 & 60.794 & 1904.5 \\
\hline$\epsilon=.35$ & 16 & 58.218 & 44.585 & 105.73 & 84.214 \\
& 32 & 60.635 & 47.596 & 104.68 & 84.659 \\
& 64 & 63.142 & 49.910 & 105.22 & 86.679 \\
& 128 & 64.920 & 51.060 & 107.28 & 89.438 \\
\hline$\epsilon=.5$ & 16 & $1.3 \times 10^{18}$ & 43.344 & $9.9 \times 10^{17}$ & 77.217 \\
& 32 & $2.6 \times 10^{17}$ & 45.345 & $4.1 \times 10^{17}$ & 78.257 \\
& 64 & $2.5 \times 10^{17}$ & 46.994 & $1.8 \times 10^{18}$ & 79.910 \\
& 128 & $6.8 \times 10^{17}$ & 48.615 & $3.9 \times 10^{18}$ & 82.596 \\
\hline$\epsilon=.65$ & 16 & 58.218 & 45.623 & 104.82 & 83.355 \\
& 32 & 60.635 & 47.543 & 102.81 & 84.815 \\
& 64 & 63.142 & 49.897 & 103.99 & 87.152 \\
& 128 & 64.920 & 51.057 & 107.17 & 89.575 \\
\hline$\epsilon=.999$ & 16 & 33.595 & 6711.6 & 55.305 & 20020.0 \\
& 32 & 35.714 & 6510.0 & 57.017 & 19216.0 \\
& 64 & 36.693 & 6423.5 & 59.119 & 19045.0 \\
& 128 & 37.188 & 6379.2 & 60.774 & 19002.0 \\
\hline
\end{tabular}

TABLE 2. Conditioning of Galerkin scheme

\begin{tabular}{ccccc|ccccc}
\hline \hline & \multicolumn{10}{c}{$\|M\|_{2}\left\|M^{-1}\right\|_{2}$} & & & & & & \\
\hline$n$ & $m=2$ & $m=3$ & $m=4$ & $m=5$ & $n$ & $m=2$ & $m=3$ & $m=4$ & $m=5$ \\
8 & 7.96 & $3.4 \times 10^{17}$ & 52.56 & $1.2 \times 10^{15}$ & 9 & 7.96 & 247.56 & 59.49 & 529.24 \\
16 & 7.96 & $3.3 \times 10^{17}$ & 64.22 & $2.4 \times 10^{15}$ & 17 & 7.96 & 909.25 & 66.76 & 1992.4 \\
32 & 7.96 & $6.6 \times 10^{17}$ & 69.03 & $5.0 \times 10^{15}$ & 33 & 7.96 & 3473.6 & 69.72 & 7706.3 \\
64 & 7.96 & $1.0 \times 10^{18}$ & 70.67 & $1.1 \times 10^{16}$ & 65 & 7.96 & 13558 & 70.85 & 30285 \\
\hline
\end{tabular}

the best conditioning of the scheme is obtained with $\epsilon$ near .5 , while taking a value close to either 0 or 1 gives larger condition numbers. The conditioning also grows very little with increasing $n$, in line with the stability results for the collocation method. Similar results are obtained with an odd number of nodes.

Now consider the Galerkin method. Table 2 contains the corresponding condition numbers in the $L_{2}$ norm. As in this case no parameter $\epsilon$ arises, the results are much easier to illustrate. Notice that this time the system is badly conditioned for an even number of nodes coupled with $m$ odd. This is probably caused by the approximation method used for calculating the scalar product. The effect is closely connected with the fact that while implementing the Galerkin method we are in reality using qualocation schemes with $\epsilon_{1}=0$. In the Galerkin case an odd $n$ also exhibits a larger conditioning for $m$ odd, compared to $m$ even, but much milder compared with the ill-conditioning of the same $m$ for $n$ even. 
Let us now illustrate the convergence of the above considered approximation methods for the Muskhelishvili equation with given right hand sides. For sake of simplicity, in these examples we use an explicit expression for the function $f_{0}$. Moreover, in all cases the curve $\Gamma$ is the unit circle, but the last example will also be considered on a family of ellipses with increasing eccentricity, to show the flexibility of the proposed methods.

Example 1. The forcing function is chosen as $f_{0}(t)=-t^{-2}$, so that an analytical solution is $x(t)=t^{2}$. These functions are defined over the unit circle, $t \in \Gamma$. The function $x(t)=t^{2}$ is just a particular solution, while the Muskhelishvili equation in this case has a family of solutions of the general form $x(t)=t^{2}+i \alpha t+\beta$, with $\alpha \in \mathbf{R}$ and $\beta \in \mathbf{C}$.

Figures 1 [2] show the error behavior of Example $1\left|x(\gamma(s))-x_{n}(\gamma(s))\right|, s \in[0,1]$, across the interval, for some selected cases of the order of splines and the collocation parameter $\epsilon$. In general, doubling $n$ provides a decrease in the error. The figures do not differ too much from each other. For the Galerkin method the error behavior is very similar and thus not reported. This once again indicates that $m$ does not play an essential role in the convergence, and that both methods behave similarly. From Figures 1.2. comparing the ratios of the heights of the vertical scales or the maxima of the functions, the latter can easily be seen to be larger than 2 , indicating superlinear convergence at the very least, in line with our theoretical results. To better assess the performance of the method, tables are included containing the empirical estimates of the convergence order $r$ from

$$
\begin{array}{cr}
r \equiv \log _{2}\left(\left\|e_{n}\right\|\right)-\log _{2}\left(\left\|e_{2 n}\right\|\right), & e_{n} \equiv x(\gamma(s))-x_{n}(\gamma(s)), \\
\hat{r} \equiv \log _{2}\left(\left\|\delta_{n}\right\|\right)-\log _{2}\left(\left\|\delta_{2 n}\right\|\right), & \delta_{n} \equiv x_{n}(\gamma(s))-x_{2 n}(\gamma(s)) .
\end{array}
$$

In Tables 3 and 4 in spite of using a higher value of $m$ in some cases, convergence does not appear to improve. In almost all cases it is barely superlinear. The periodicity of the problem might explain the good performance for the choice $m=2$. Thus the order of the error does not seem to be influenced by the choice of the order $m$ of the splines. Theoretically, convergence is determined indeed only by $n^{-1}$. The error might be improved by using higher order quadratures for the calculation of the corresponding integrals.

The right hand sides in the next examples are arbitrarily chosen. Convergence of the numerical schemes follows comparing runs with an increasing number of grid points, $n$. As the analytic solution is not available for these examples, we show the graphs of the solutions $x_{n}(t), t \in \Gamma$, for various increasing values of $n$. The figures below show convergence of the algorithms, as the former become closer the higher $n$ is. This happens both with different choices of the order of the splines as well as by using different methods for their calculations. When the analytic solution is not available, these calculations also validate the code since the same solution is always reproduced.

Example 2. In this case we chose $f_{0}(t)=\frac{i}{t} \overline{\cos (t)}-\frac{1}{t}$.

Example 3. Here we chose $f_{0}(t)=\frac{t^{3}}{t^{2}+2}$. 
TABLE 3. Convergence order of collocation scheme on Example 1

\begin{tabular}{|c|c|c|c|c|c|c|c|c|}
\hline & $m=2$ & $\epsilon=0.1$ & & & $m=2$ & $\epsilon=0.45$ & & \\
\hline$n$ & $\left\|e_{n}\right\|_{\infty}$ & $r$ & $\left\|\delta_{n}\right\|_{\infty}$ & $\hat{r}$ & $\left\|e_{n}\right\|_{\infty}$ & $r$ & $\left\|\delta_{n}\right\|_{\infty}$ & $\hat{r}$ \\
\hline 4 & 1.03 & - & - & - & 9.89 & - & - & - \\
\hline 8 & $2.24 \times 10^{-1}$ & 2.20 & 1.07 & - & $4.08 \times 10^{-1}$ & 4.60 & 9.85 & - \\
\hline 16 & $5.10 \times 10^{-2}$ & 2.13 & $2.39 \times 10^{-1}$ & 2.17 & $8.15 \times 10^{-2}$ & 2.32 & $3.27 \times 10^{-1}$ & 4.91 \\
\hline 32 & $1.24 \times 10^{-2}$ & 2.04 & $5.50 \times 10^{-2}$ & 2.12 & $1.94 \times 10^{-2}$ & 2.07 & $6.21 \times 10^{-2}$ & 2.39 \\
\hline 64 & $3.08 \times 10^{-3}$ & 2.01 & $1.34 \times 10^{-2}$ & 2.03 & $4.79 \times 10^{-3}$ & 2.02 & $1.46 \times 10^{-2}$ & 2.09 \\
\hline \multirow[t]{2}{*}{128} & $7.70 \times 10^{-4}$ & 2.00 & $3.33 \times 10^{-3}$ & 2.01 & $1.19 \times 10^{-3}$ & 2.00 & $3.60 \times 10^{-3}$ & 2.02 \\
\hline & $m=3$ & $\epsilon=0.7$ & & & $m=4$ & $\epsilon=0.45$ & & \\
\hline$n$ & $\left\|e_{n}\right\|_{\infty}$ & $r$ & $\left\|\delta_{n}\right\|_{\infty}$ & $\tilde{r}$ & $\left\|e_{n}\right\|_{\infty}$ & $r$ & $\left\|\delta_{n}\right\|_{\infty}$ & $\hat{r}$ \\
\hline 4 & 2.18 & - & - & - & $1.98 \times 10^{1}$ & - & - & - \\
\hline 8 & $2.71 \times 10^{-1}$ & 3.01 & 2.01 & - & $4.57 \times 10^{-1}$ & 5.44 & $1.94 \times 10^{1}$ & - \\
\hline 16 & $7.62 \times 10^{-2}$ & 1.83 & $2.07 \times 10^{-1}$ & 3.28 & $1.49 \times 10^{-1}$ & 1.62 & $3.36 \times 10^{-1}$ & 5.85 \\
\hline 32 & $3.28 \times 10^{-2}$ & 1.22 & $4.50 \times 10^{-2}$ & 2.20 & $6.64 \times 10^{-2}$ & 1.17 & $8.66 \times 10^{-2}$ & 1.96 \\
\hline 64 & $1.58 \times 10^{-2}$ & 1.06 & $1.71 \times 10^{-2}$ & 1.40 & $3.18 \times 10^{-2}$ & 1.06 & $3.50 \times 10^{-2}$ & 1.31 \\
\hline 128 & $7.76 \times 10^{-3}$ & 1.02 & $8.02 \times 10^{-3}$ & 1.09 & $1.56 \times 10^{-2}$ & 1.03 & $1.62 \times 10^{-2}$ & 1.11 \\
\hline
\end{tabular}

TABLE 4. Convergence order of Galerkin scheme on Example 1

\begin{tabular}{|c|c|c|c|c|c|c|c|c|}
\hline & $m=2$ & & & & $m=3$ & & & \\
\hline$n$ & $\left\|e_{n}\right\|_{2}$ & $r$ & $\left\|\delta_{n}\right\|_{2}$ & $\tilde{r}$ & $\left\|e_{n}\right\|_{2}$ & $r$ & $\left\|\delta_{n}\right\|_{2}$ & $\hat{r}$ \\
\hline 5 & 1.29 & - & - & - & $2.20 \mathrm{e}+00$ & - & - & - \\
\hline 9 & $3.28 \times 10^{-1}$ & 1.98 & $7.45 \times 10^{-1}$ & - & $2.42 \mathrm{e}-01$ & 3.18 & $1.47 \mathrm{e}+00$ & - \\
\hline 17 & $6.93 \times 10^{-2}$ & 2.24 & $1.85 \times 10^{-1}$ & 2.01 & $6.83 \times 10^{-2}$ & 1.82 & $1.13 \times 10^{-1}$ & 3.70 \\
\hline 33 & $1.06 \times 10^{-2}$ & 2.71 & $4.10 \times 10^{-2}$ & 2.18 & $2.29 \times 10^{-2}$ & 1.58 & $2.65 \times 10^{-2}$ & 2.09 \\
\hline 65 & $2.54 \times 10^{-3}$ & 2.06 & $5.51 \times 10^{-3}$ & 2.89 & $8.02 \times 10^{-3}$ & 1.52 & $8.35 \times 10^{-3}$ & 1.67 \\
\hline \multirow[t]{2}{*}{129} & $4.35 \times 10^{-4}$ & 2.54 & $1.46 \times 10^{-3}$ & 1.91 & $2.83 \times 10^{-3}$ & 1.51 & $2.87 \times 10^{-3}$ & 1.54 \\
\hline & $m=4$ & & & & $m=5$ & & & \\
\hline$n$ & $\left\|e_{n}\right\|_{2}$ & $r$ & $\left\|\delta_{n}\right\|_{2}$ & $\tilde{r}$ & $\left\|e_{n}\right\|_{2}$ & $r$ & $\left\|\delta_{n}\right\|_{2}$ & $\hat{r}$ \\
\hline 5 & 2.56 & - & - & - & $8.25 \mathrm{e}+00$ & - & - & - \\
\hline 9 & $4.83 \times 10^{-1}$ & 2.40 & 1.64 & - & $8.51 \times 10^{-1}$ & 3.28 & 5.74 & - \\
\hline 17 & $1.40 \times 10^{-1}$ & 1.79 & $2.33 \times 10^{-1}$ & 2.82 & $2.21 \times 10^{-1}$ & 1.94 & $4.61 \times 10^{-1}$ & 3.64 \\
\hline 33 & $4.66 \times 10^{-2}$ & 1.59 & $5.61 \times 10^{-2}$ & 2.05 & $7.15 \times 10^{-2}$ & 1.63 & $9.38 \times 10^{-2}$ & 2.30 \\
\hline 65 & $1.62 \times 10^{-2}$ & 1.53 & $1.73 \times 10^{-2}$ & 1.70 & $2.45 \times 10^{-2}$ & 1.55 & $2.71 \times 10^{-2}$ & 1.79 \\
\hline 129 & $5.67 \times 10^{-3}$ & 1.51 & $5.82 \times 10^{-3}$ & 1.57 & $8.56 \times 10^{-3}$ & 1.52 & $8.90 \times 10^{-3}$ & 1.61 \\
\hline
\end{tabular}

In Tables 5 and 6 we give the empirical orders of convergence $\hat{r}$ for both collocation and Galerkin methods. On these two examples for collocation for $m=3$ we obtain superlinear convergence, for the two values of $\epsilon$ chosen, but for $m=2$ and $m=4$ we find more than quadratic convergence again independently of $\epsilon$. Similar results hold for the Galerkin case.

The Figures 3 8 exhibit the behavior of the algorithms on these examples. Note that the first frame in every figure shows that each algorithm begins with a somewhat different first approximation of the solution, which however is automatically corrected the larger the values of $n$. An instance of this sort is seen in Figure 3 , where the initial "broken line" approximation is smoothed out, and in Figure 4 It is also apparent that choosing $n$ even or odd does not influence the later stages of the computations. 
TABle 5. Convergence order of collocation scheme

\begin{tabular}{ccc|ccc}
\hline \hline Example 2 & $m=3$ & $\epsilon=0.1$ & Example 3 & $m=4$ & $\epsilon=0.3$ \\
$n$ & $\left\|\delta_{n}\right\|_{\infty}$ & $\hat{r}$ & $n$ & $\left\|\delta_{n}\right\|_{\infty}$ & $\hat{r}$ \\
\hline 4 & - & - & 4 & - & - \\
8 & 1.67 & - & 8 & 1.21 & - \\
16 & $5.11 \times 10^{-1}$ & 1.71 & 16 & $8.55 \times 10^{-1}$ & 0.50 \\
32 & $4.75 \times 10^{-2}$ & 3.43 & 32 & $3.52 \times 10^{-1}$ & 1.28 \\
64 & $1.33 \times 10^{-2}$ & 1.83 & 64 & $5.07 \times 10^{-2}$ & 2.80 \\
128 & $5.77 \times 10^{-3}$ & 1.21 & 128 & $5.97 \times 10^{-3}$ & 3.09 \\
\hline Example 2 & $m=3$ & $\epsilon=0.6$ & Example 3 & $m=2$ & $\epsilon=0.6$ \\
$n$ & $\left\|\delta_{n}\right\|_{\infty}$ & $\hat{r}$ & $n$ & $\left\|\delta_{n}\right\|_{\infty}$ & $\hat{r}$ \\
\hline 4 & - & - & 4 & - & - \\
8 & 1.01 & - & 8 & 1.76 & - \\
16 & $3.44 \times 10^{-1}$ & 1.55 & 16 & 1.93 & -0.14 \\
32 & $4.82 \times 10^{-2}$ & 2.84 & 32 & $9.58 \times 10^{-1}$ & 1.01 \\
64 & $1.41 \times 10^{-2}$ & 1.77 & 64 & $1.83 \times 10^{-1}$ & 2.39 \\
128 & $5.88 \times 10^{-3}$ & 1.26 & 128 & $3.30 \times 10^{-2}$ & 2.47 \\
\hline
\end{tabular}

TABLE 6. Convergence order of Galerkin scheme

\begin{tabular}{ccc|ccc}
\hline \hline Example 2 & $m=3$ & & Example 3 & $m=4$ & \\
$n$ & $\left\|\delta_{n}\right\|_{2}$ & $\hat{r}$ & $\mathrm{n}$ & $\left\|\delta_{n}\right\|_{2}$ & $\hat{r}$ \\
\hline 5 & - & - & 5 & - & - \\
9 & 7.06 & - & 9 & 4.90 & - \\
17 & $8.50 \times 10^{-1}$ & 3.05 & 17 & 1.47 & 1.74 \\
33 & $7.04 \times 10^{-2}$ & 3.59 & 33 & $2.64 \times 10^{-1}$ & 2.48 \\
65 & $1.96 \times 10^{-2}$ & 1.84 & 65 & $2.75 \times 10^{-2}$ & 3.26 \\
129 & $6.40 \times 10^{-3}$ & 1.61 & 129 & $6.72 \times 10^{-3}$ & 2.03 \\
\hline
\end{tabular}

TABLe 7. Conditioning in elliptic case for $b=2$

\begin{tabular}{c|ccccc}
\hline \hline & & $a=6$ & $a=10$ & $a=18$ & $a=25$ \\
\hline collocation & $m=2$ & $5.1 \times 10^{2}$ & $8.3 \times 10^{2}$ & $1.5 \times 10^{3}$ & $1.9 \times 10^{3}$ \\
$\|M\|_{\infty}\left\|M^{-1}\right\|_{\infty}$ & $m=3$ & $5.6 \times 10^{2}$ & $9.2 \times 10^{2}$ & $1.9 \times 10^{3}$ & $3.3 \times 10^{3}$ \\
$n=128$ & $m=4$ & $7.1 \times 10^{2}$ & $1.3 \times 10^{3}$ & $3.3 \times 10^{3}$ & $7.2 \times 10^{3}$ \\
$\epsilon=.25$ & $m=5$ & $8.4 \times 10^{2}$ & $1.6 \times 10^{3}$ & $4.8 \times 10^{3}$ & $1.3 \times 10^{4}$ \\
\hline Galerkin $\|M\|_{2}\left\|M^{-1}\right\|_{2}$ & $m=2$ & $1.2 \times 10^{2}$ & $3.0 \times 10^{2}$ & $9.9 \times 10^{2}$ & $1.9 \times 10^{3}$ \\
$n=129$ & $m=4$ & $8.0 \times 10^{2}$ & $2.2 \times 10^{3}$ & $7.5 \times 10^{3}$ & $1.8 \times 10^{4}$ \\
\hline
\end{tabular}

In this case, Figures 3 and 4 are evaluated for $m=2$ and $m=4$ and with different $\epsilon$ and an even $n$, while Figures $[5$ and 6 for $m=3$ and $m=4$ with an odd $n$. In all cases for the larger values of $n$ the same solutions are obtained.

In Figures 7 and 8 the two methods are used with different parity of $n$, but with the same $m=3$, still to obtain the same solution. 
Example 4. Our final choice is $f_{0}(t)=\frac{\cos (t)-i t^{2}}{\sin (t)}$.

The previous examples were only concerned with the circular domain. For such a domain the biharmonic problem as well as the Muskhelishvili equation were solved by approximation methods based on the Fourier series expansion [1], [2], 17. However if the boundary differs from the circle the methods mentioned may experience difficulties, as mentioned in 1], even for ellipses with small eccentricity. The reason is that they require conformal mappings from the unit disk. The methods we propose here are free from such problems, as evidenced by Figures 9, 16. In the latter we approximate the solution of the Muskhelishvili equation with the same right hand side of Example 4, but formulated on the unit circle and on ellipses with increasing eccentricity. It is apparent that the solutions differ for different values of this parameter, but the algorithms are still stable and pick up the fine details of each solution, provided that a sufficient number of nodes is used. In addition, one can note a remarkable transformation of the solution caused by the changing of the contour. As a final check, the Galerking method has also been used on Example 4, running it with different $m$ but with the same eccentricity, to compare the results of the collocation method. The reader should compare Figures 11 and 12, 13 and 14, [15] and 16 respectively. We also remark that the condition numbers for the larger values of $n$ in these cases are essentially the same, provided $\epsilon$ is not close to the value 0.5 , independently of the number of nodes, up to $n=512$ and of the order of the splines used. Specifically keeping $b=2$, the condition number is evaluated in the following Table 7 for both methods; however as we already remarked that an odd $m$ makes the Galerkin method ill-conditioned, we do not report these results. Conditioning grows mildly with both methods for an increasing eccentricity of the contour. 

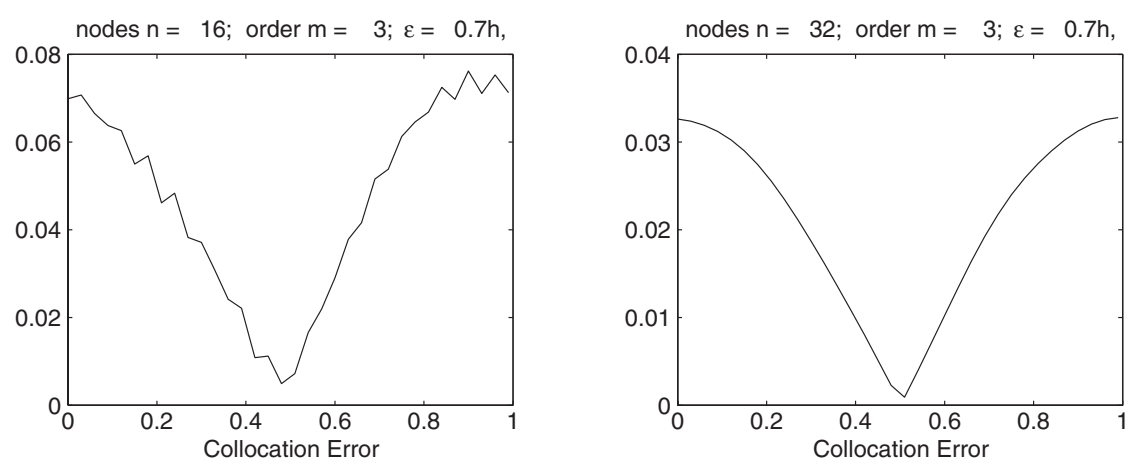

nodes $n=64 ;$ order $m=3 ; \varepsilon=0.7 \mathrm{~h}$
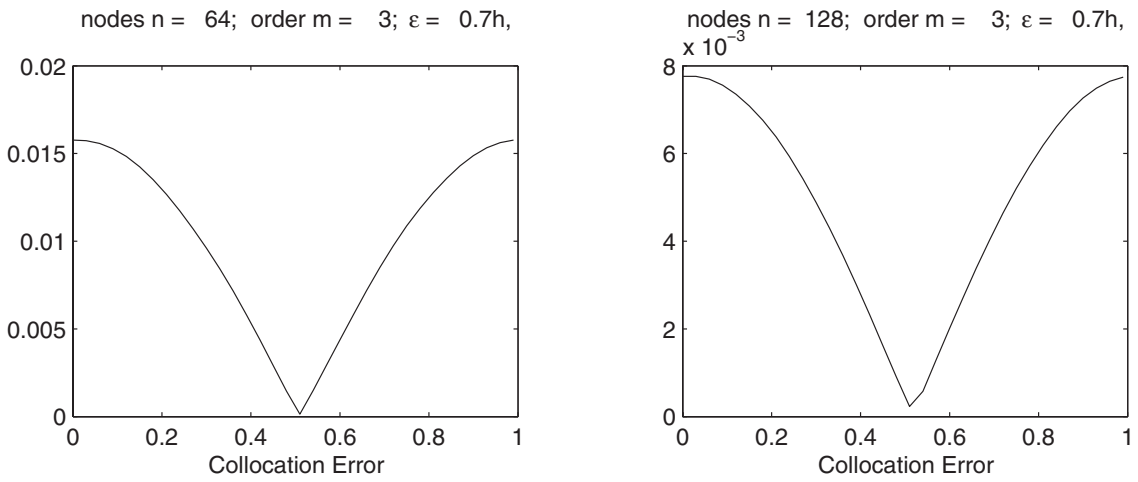

Figure 1. Collocation error for Example $1,\left|x(\gamma(s))-x_{n}(\gamma(s))\right|, s \in[0,1]$
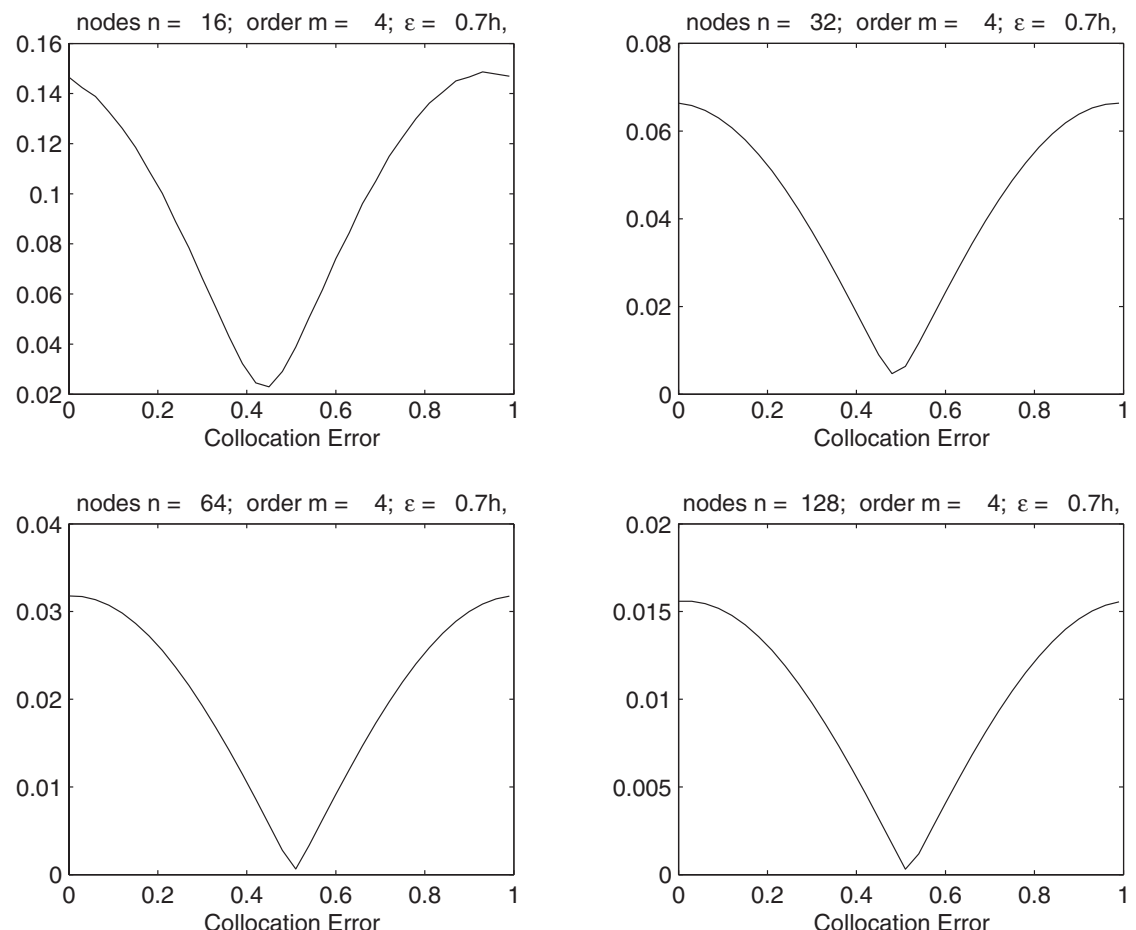

Figure 2. Collocation error for Example $1,\left|x(\gamma(s))-x_{n}(\gamma(s))\right|, s \in[0,1]$ 

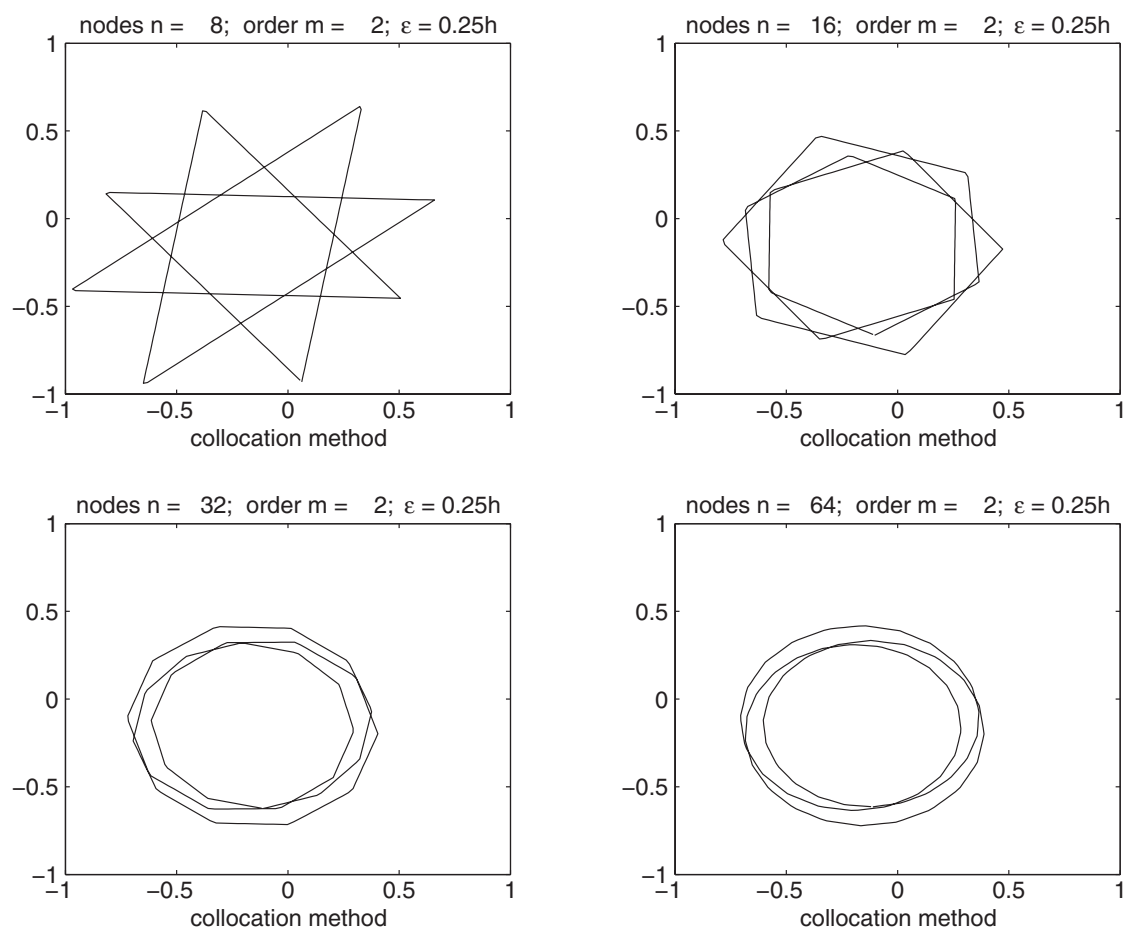

Figure 3. Example 2 collocation solution $x_{n}(t), t \in \Gamma$
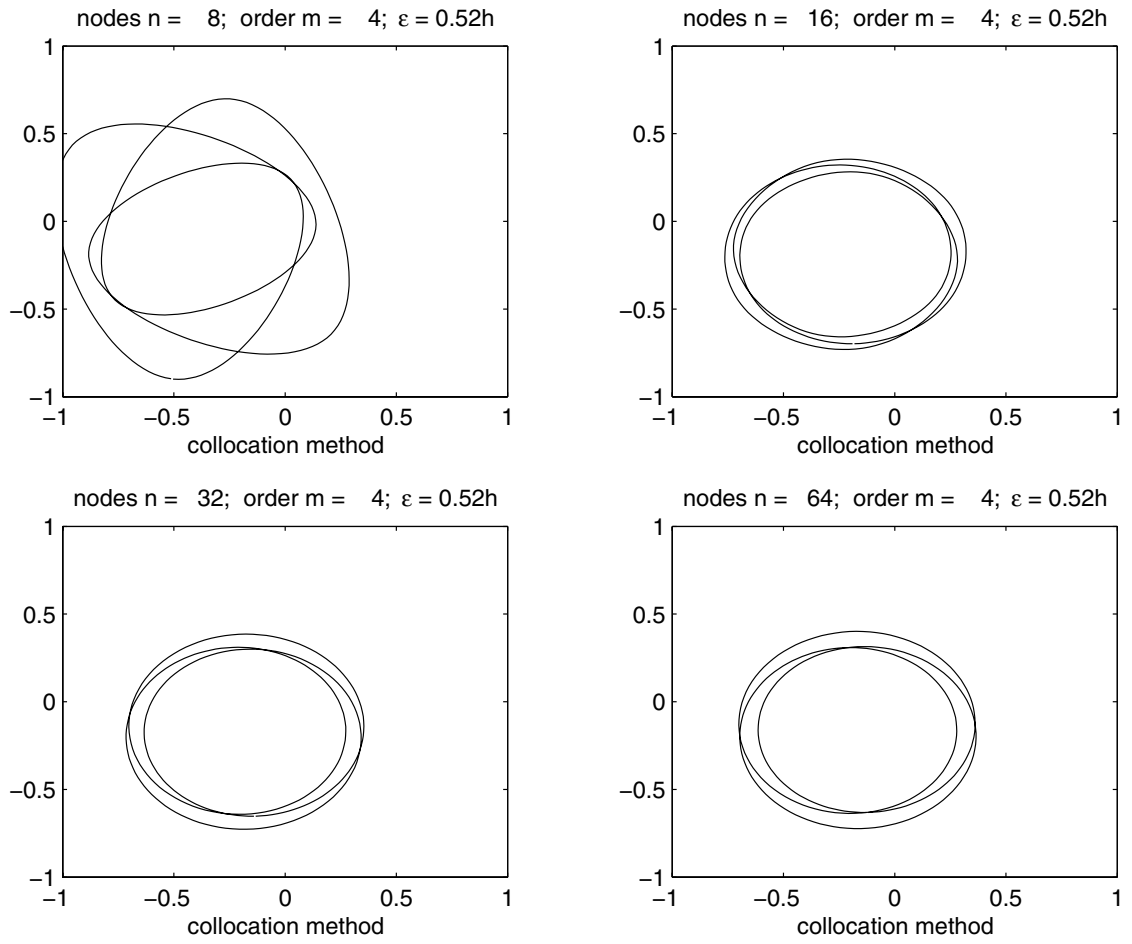

Figure 4. Example 2 collocation solution $x_{n}(t), t \in \Gamma$ 

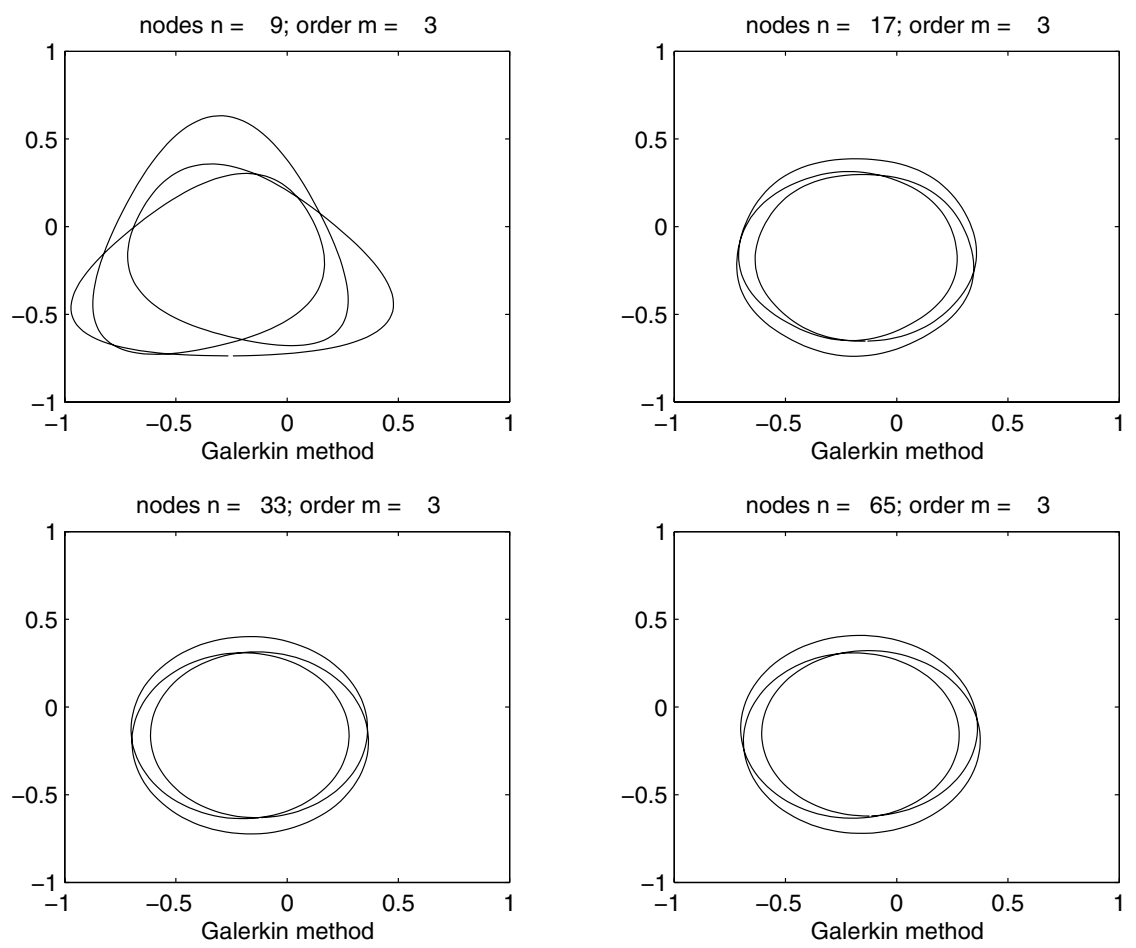

Figure 5. Example 2 Galerkin solution $x_{n}(t), t \in \Gamma$
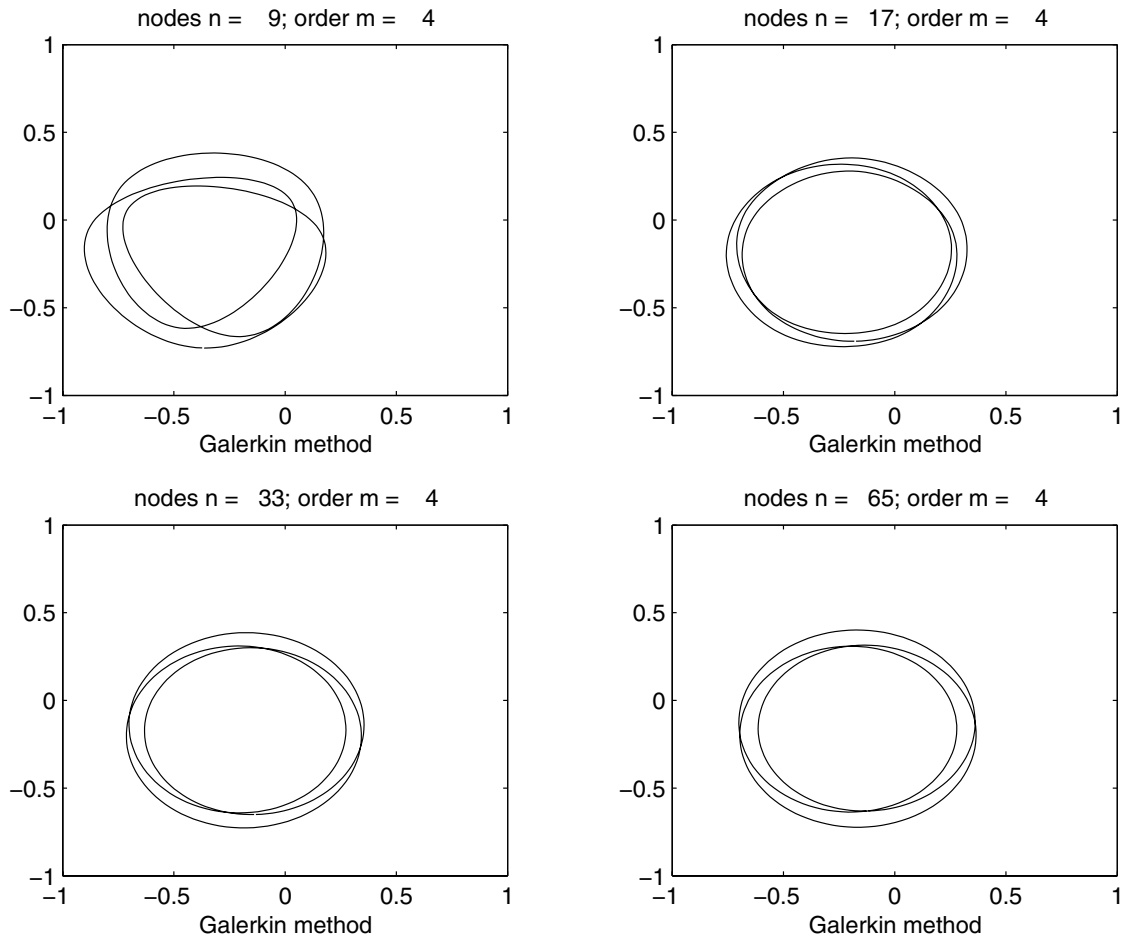

Figure 6. Example 2 Galerkin solution $x_{n}(t), t \in \Gamma$ 

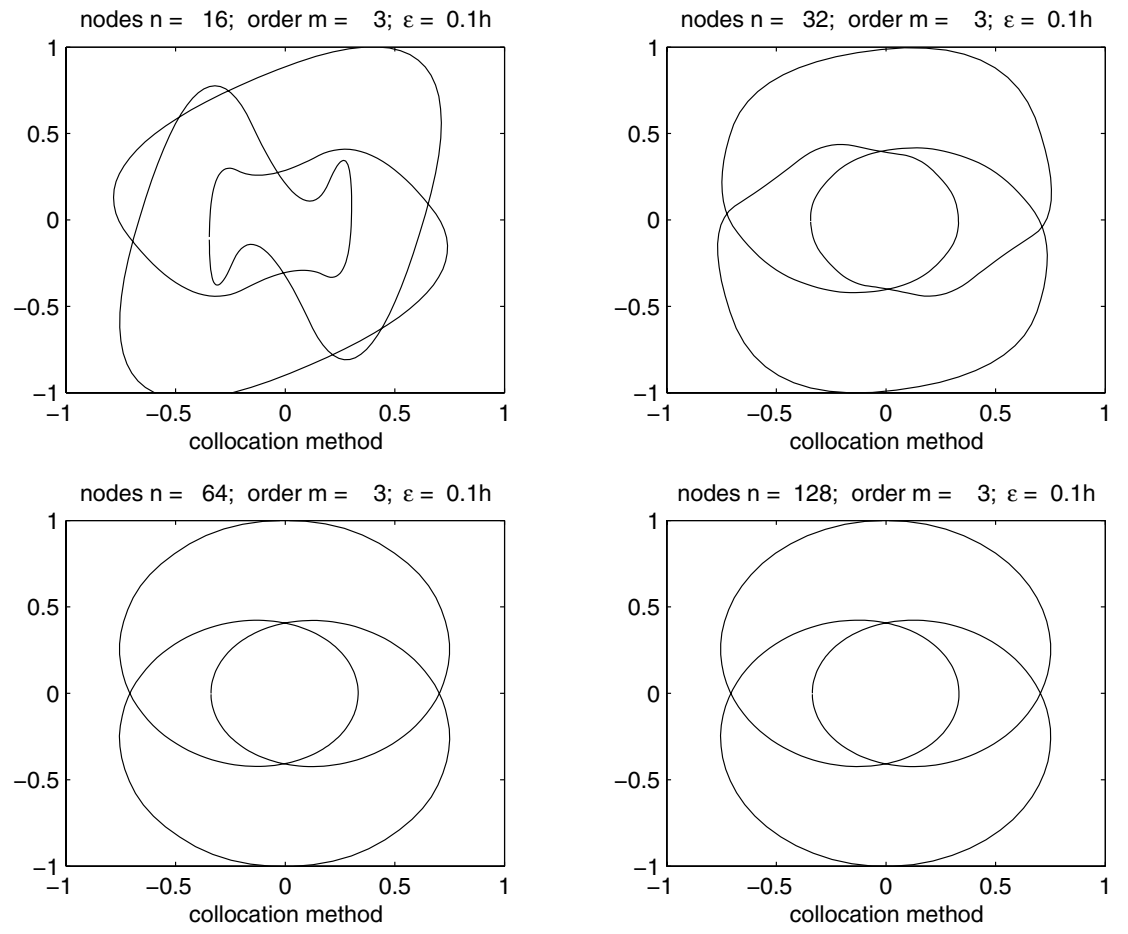

FIgURE 7. Example 3 collocation solution $x_{n}(t), t \in \Gamma$
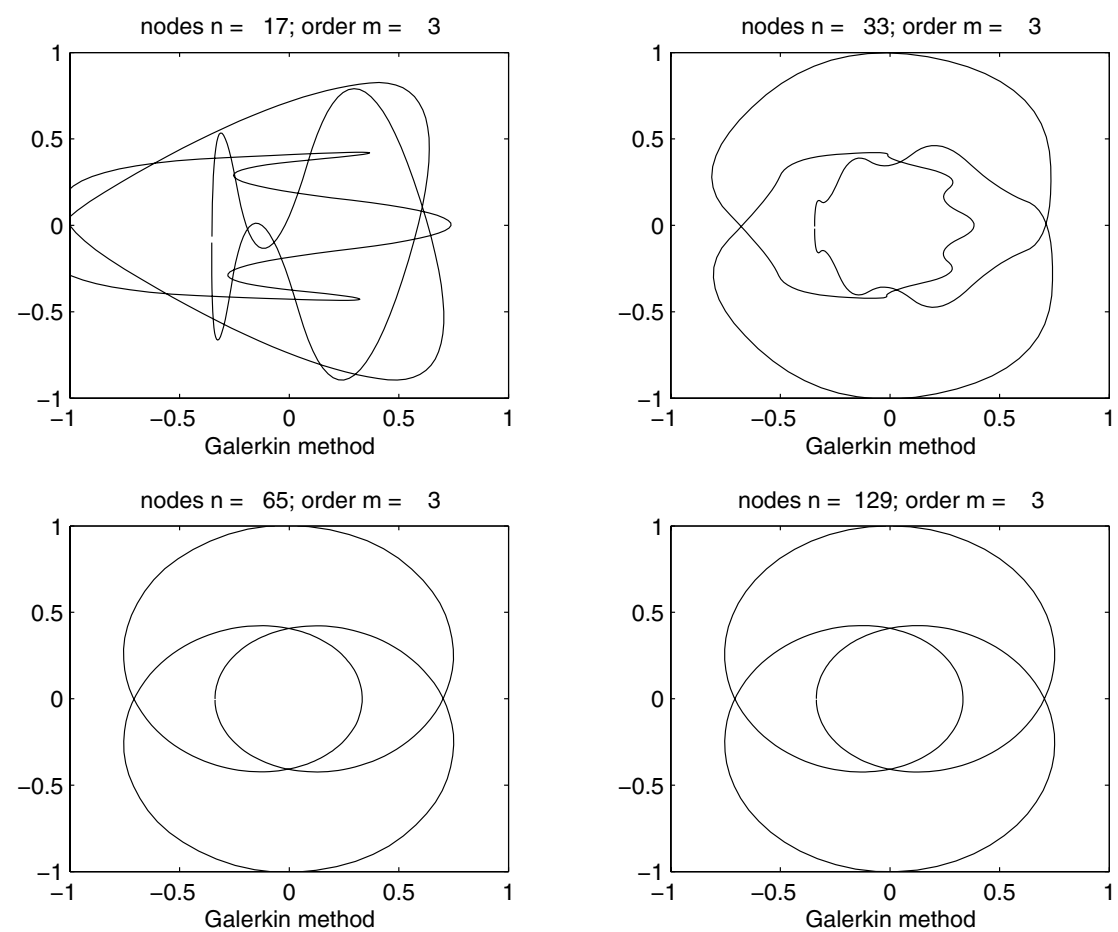

Figure 8. Example 3 Galerkin solution $x_{n}(t), t \in \Gamma$ 

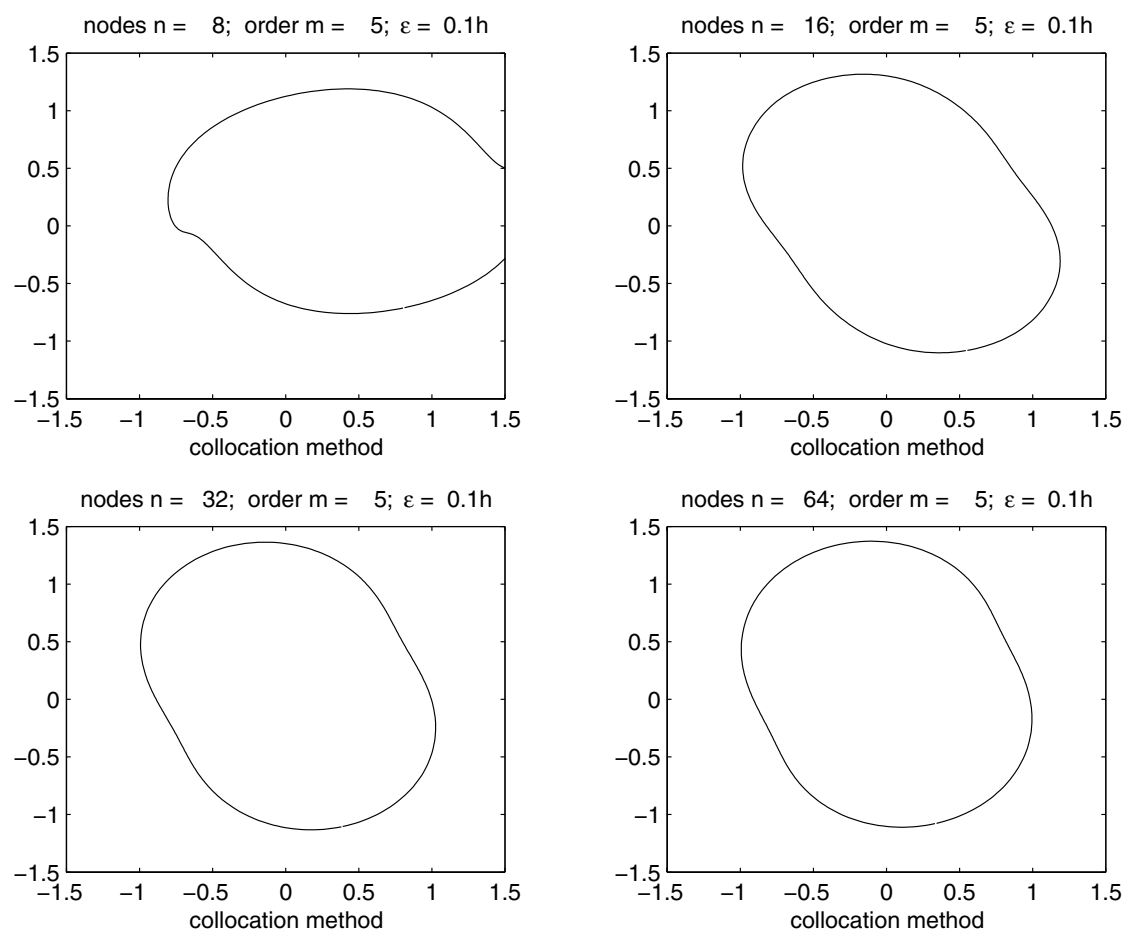

Figure 9. Example 4 collocation solution $x_{n}(t), t \in \Gamma$, the unit circle
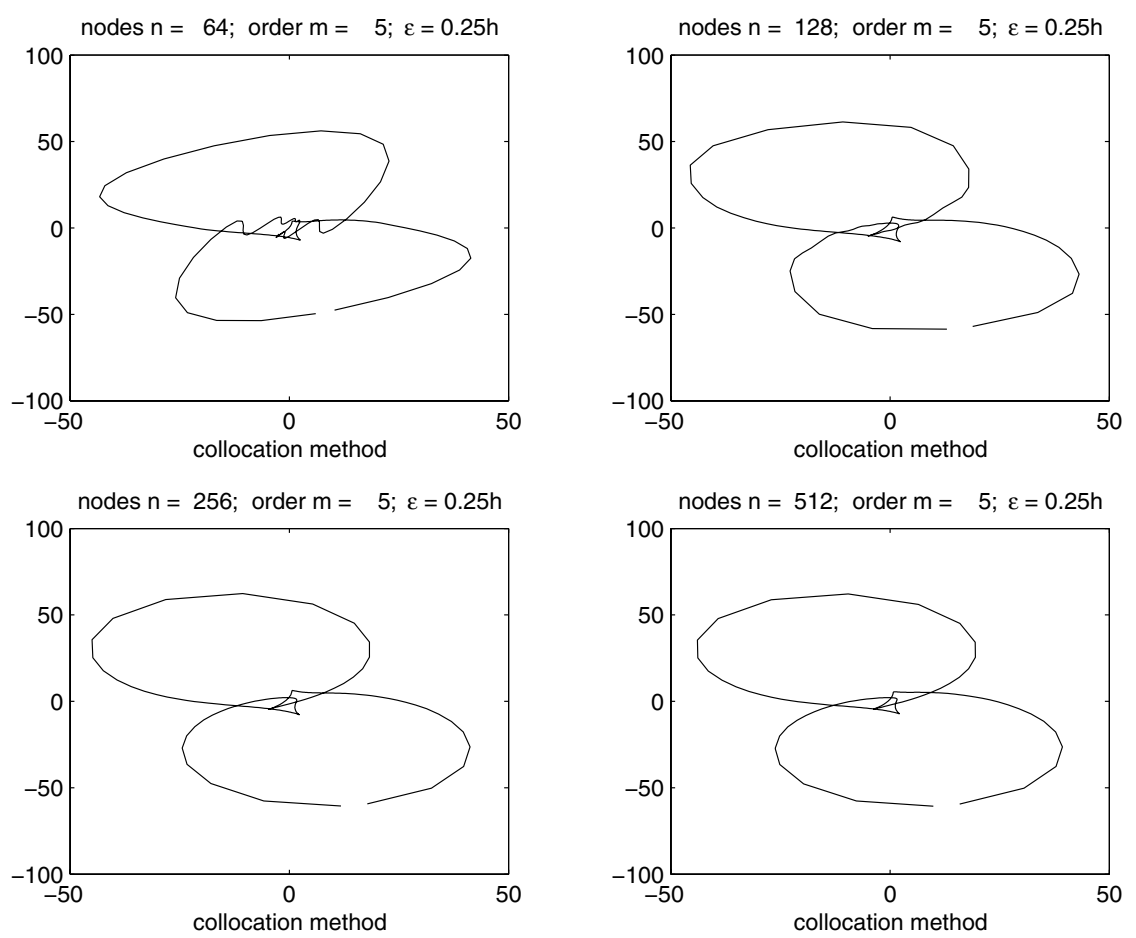

FiguRE 10. Example 4 collocation solution $x_{n}(t), t \in \Gamma$, where $\Gamma$ is now the ellipse with semiaxes $a=3, b=2$ 

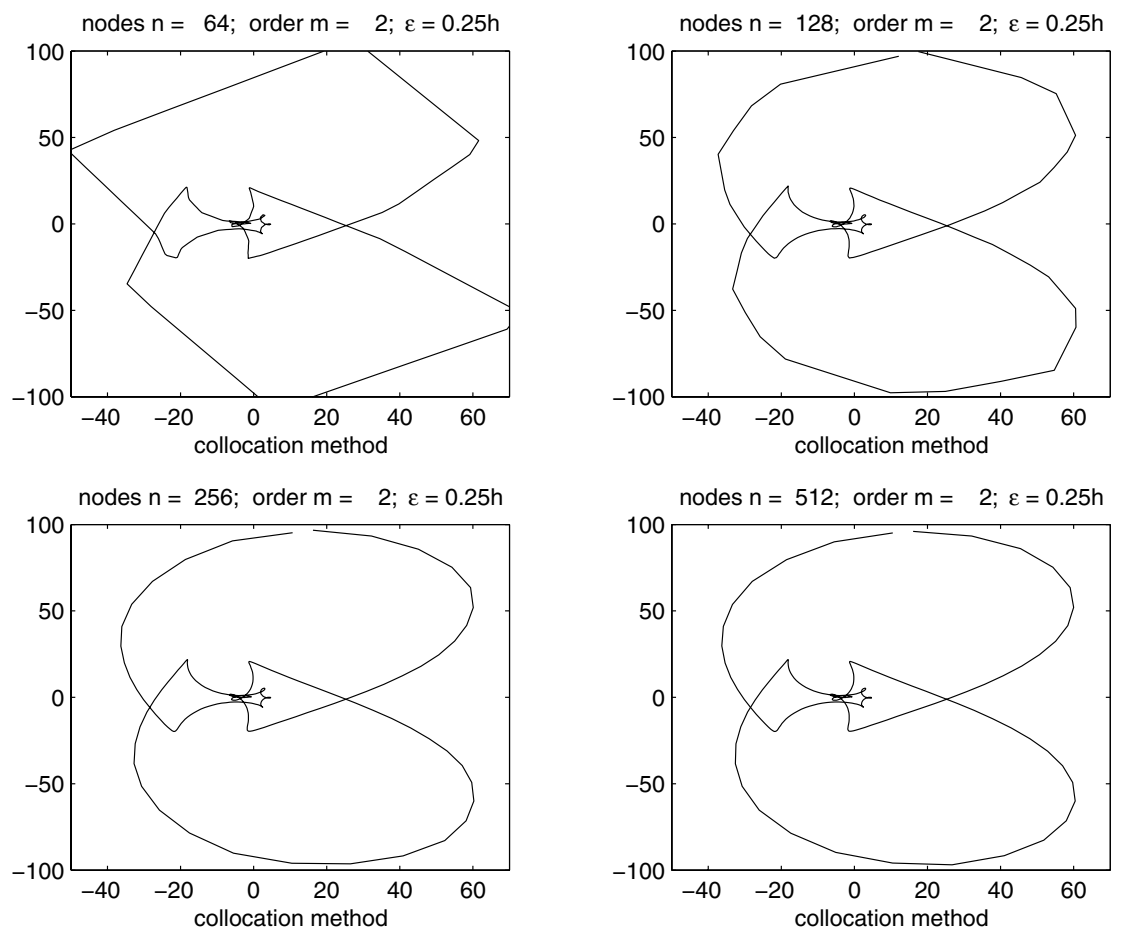

Figure 11. Example 4 collocation solution $x_{n}(t), t \in \Gamma$, where $\Gamma$ is now the ellipse with semiaxes $a=6, b=2$
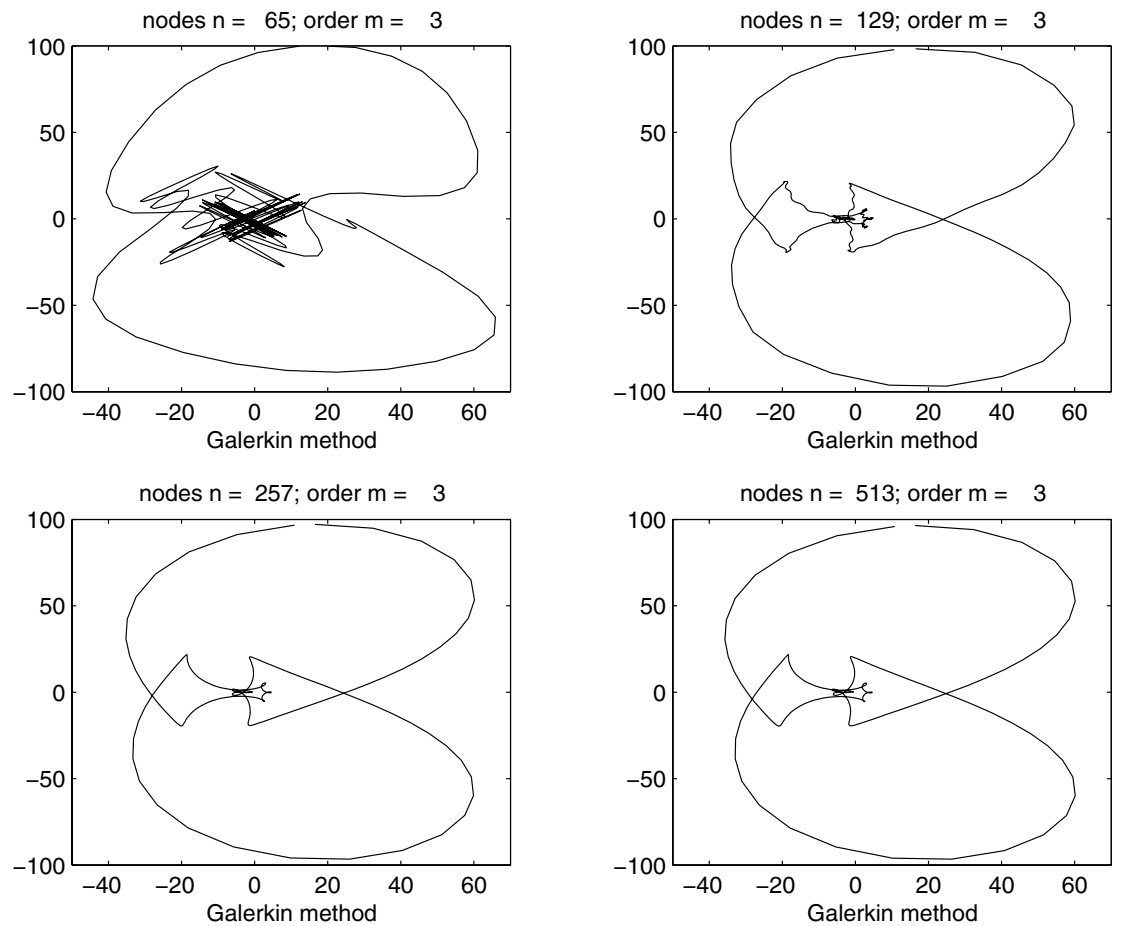

Figure 12. Example 4 Galerkin solution $x_{n}(t), t \in \Gamma$, where $\Gamma$ is now the ellipse with semiaxes $a=6, b=2$ 

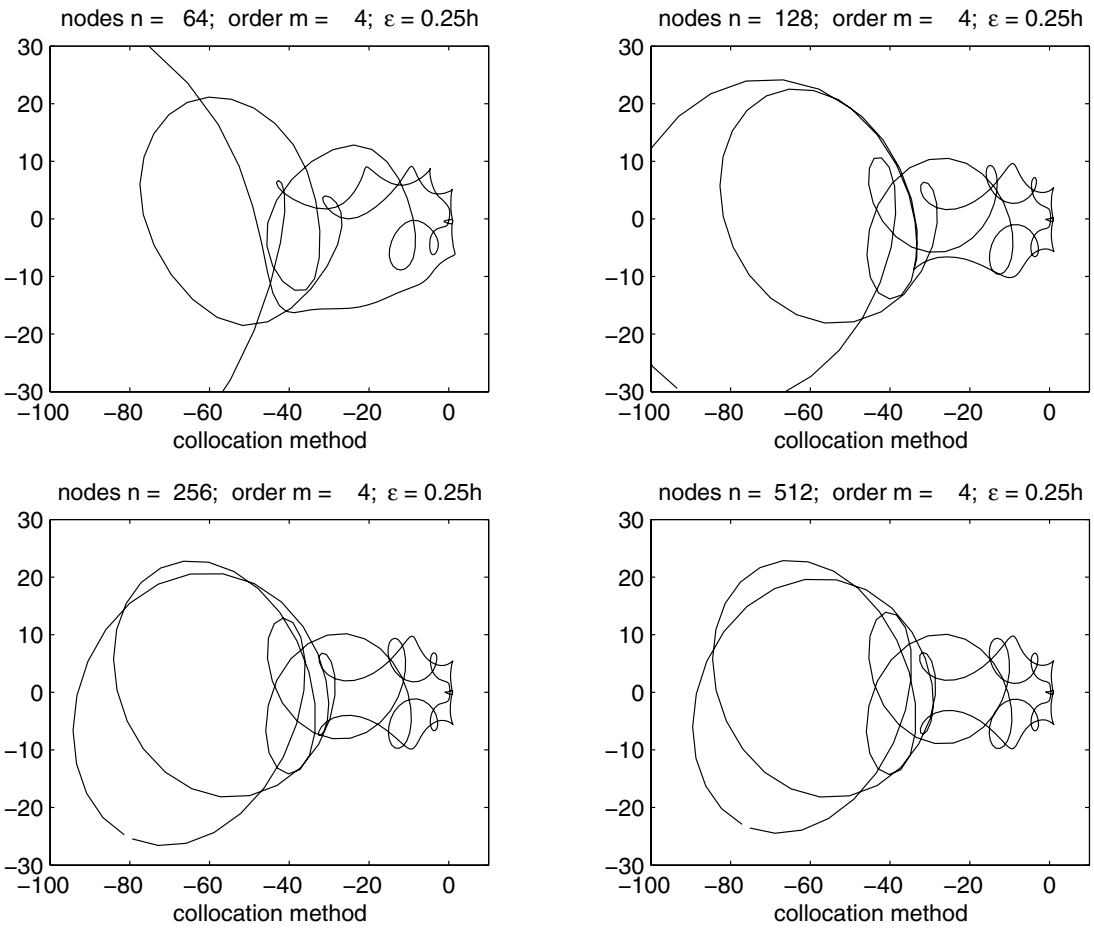

Figure 13. Example 4 collocation solution $x_{n}(t), t \in \Gamma$, where $\Gamma$ is now the ellipse with semiaxes $a=10, b=2$
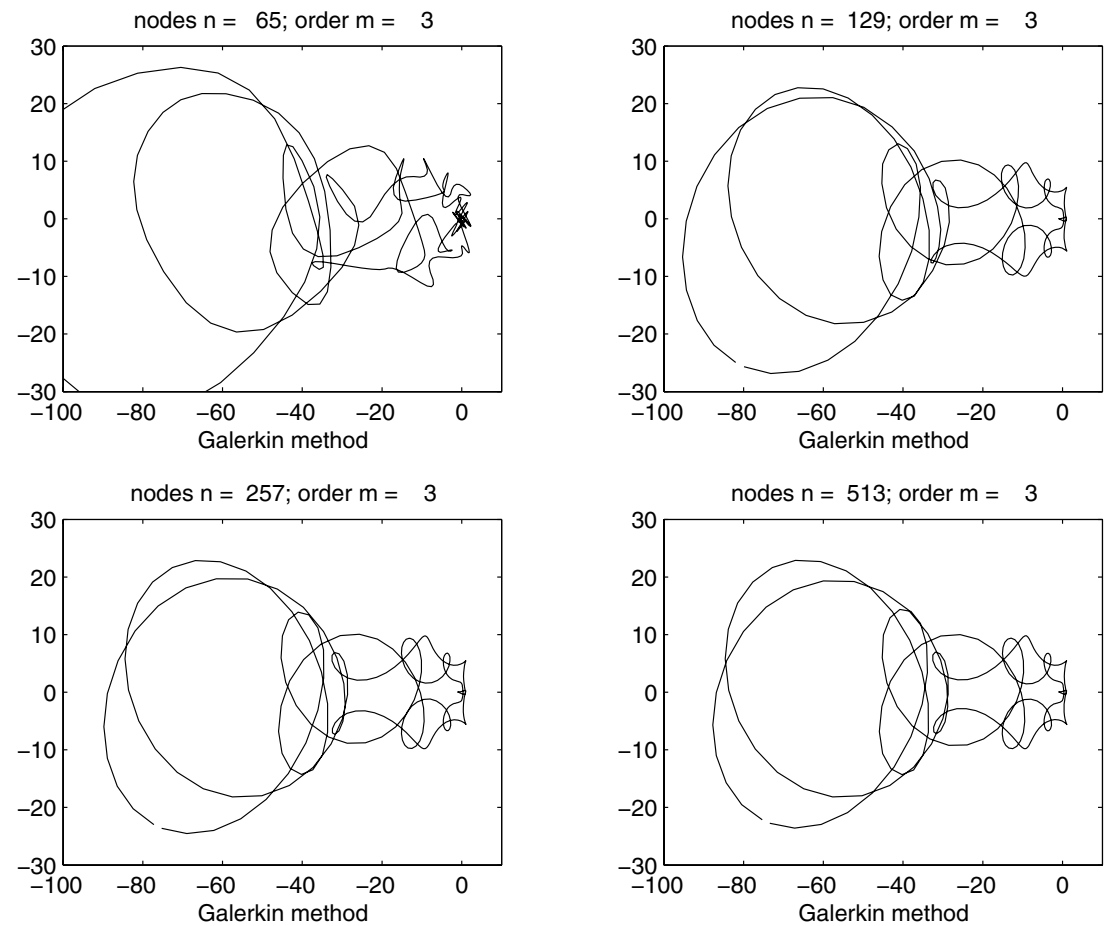

Figure 14. Example 4 Galerkin solution $x_{n}(t), t \in \Gamma$, where $\Gamma$ is now the ellipse with semiaxes $a=10, b=2$ 

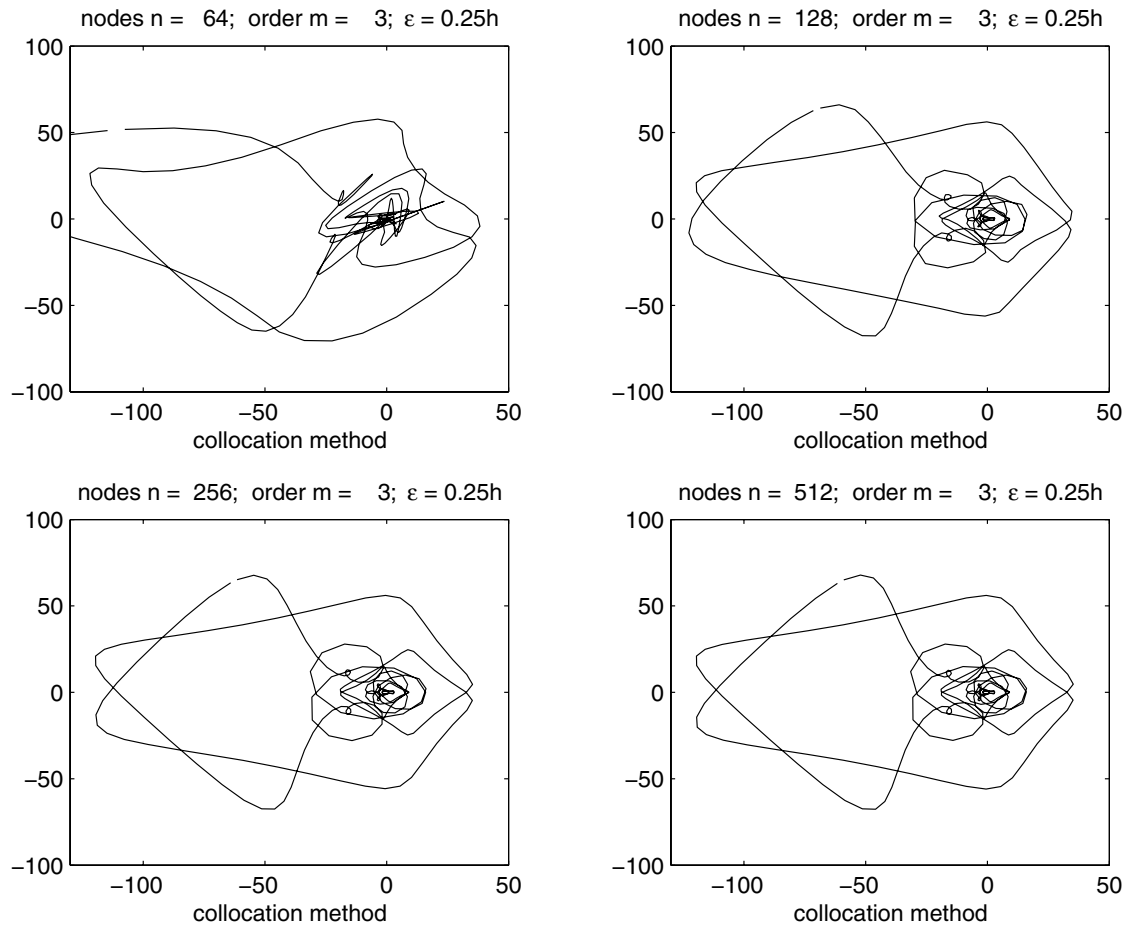

Figure 15. Example 4 collocation solution $x_{n}(t), t \in \Gamma$, where $\Gamma$ is now the ellipse with semiaxes $a=18, b=2$
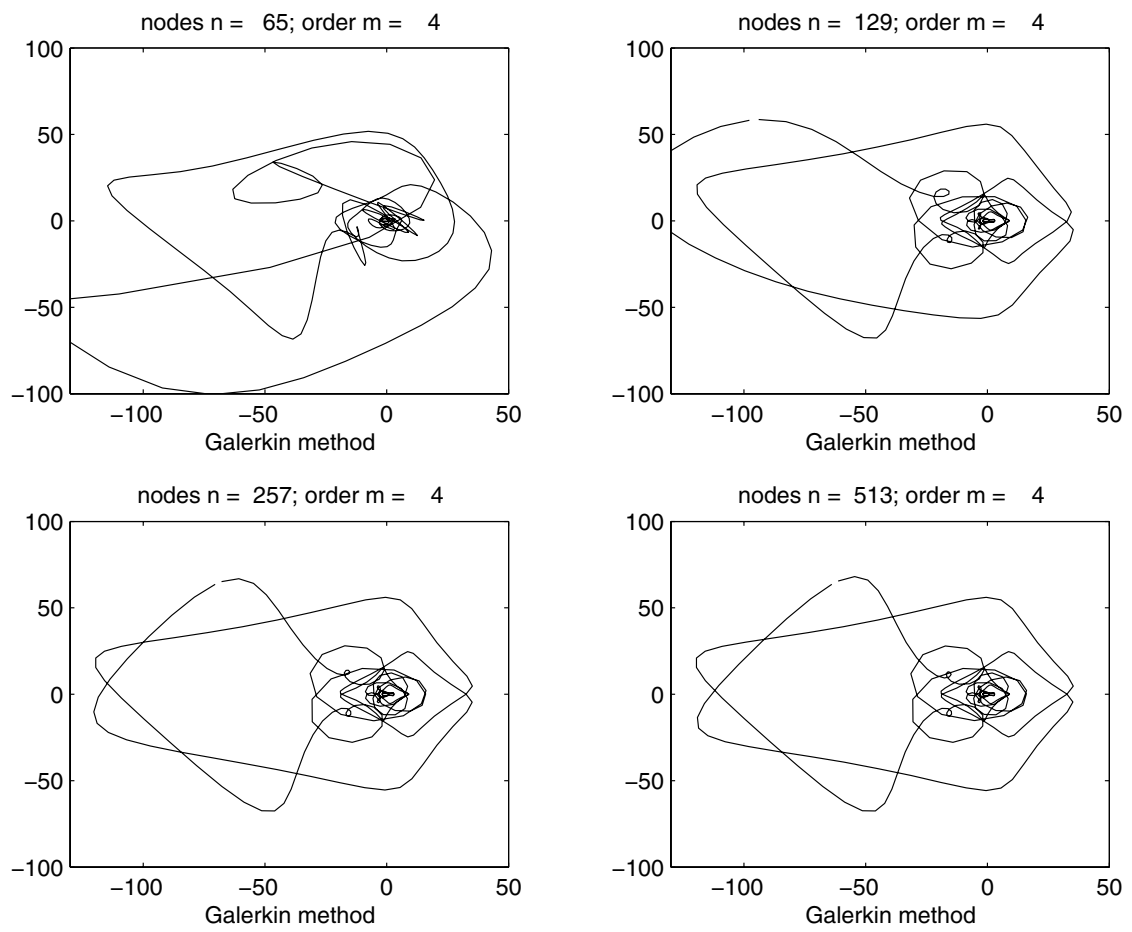

Figure 16. Example 4 Galerkin solution $x_{n}(t), t \in \Gamma$, where $\Gamma$ is now the ellipse with semiaxes $a=18, b=2$ 


\section{REFERENCES}

[1] R.H. Chan, T.K. DeLillo, M.A. Horn, Numerical solution of the biharmonic equation by conformal mapping, SIAM J. Sci. Comput. 18 (1997), p. 1571-1582. MR1480625 (98i:30005)

[2] R.H. Chang, T.K. DeLillo, M.A. Horn, Superlinear convergence estimates for a conjugate gradient method for the biharmonic equation, SIAM J. Sci. Comput. 19 (1998), p. 139-147. MR.1616882(99e:65145)

[3] J.M. Chuang, S.Z. Hu, Numerical computation of Muskhelishvili's integral equation in plane elasticity, J. Comput. Appl. Math. 66 (1996), p. 123-138. MR1393724 (97g:65275)

[4] M. Costabel and J. Saranen, Boundary element analysis of a direct method for a biharmonic Dirichlet problem, in Operator Theory: Advances and Applications, 41, p. 77-95. Birkhäuser, 1989. MR.1038333 (90m:65195)

[5] M. Costabel, J. Saranen, and I. Lusikka, Comparison of three boundary element approaches for the solution of the clamped plate problem, in C. A. Brebbia, Editor, Boundary Elements, Vol. IX, p. 19-34. Springer, 1989. MR965334 (89j:73004)

[6] M. Costabel and M. Dauge, Invertibility of the biharmonic single layer potential operator, Integr. Equat. Oper. Th. 24 (1996) 46 - 67. MR.1366540 (96m:35068)

[7] P. Davis, P. Rabinowitz, Methods of Numerical Integration, Second Edition, Academic Press, 1984. MR760629 (86d:65004)

[8] V.D. Didenko, B. Silbermann, On stability of approximation methods for the Muskhelishvili equation, J. Comput. Appl. Math. 146/2 (2002), p. 419-441. MR 1925971 (2003h:65178)

[9] V.D. Didenko, B. Silbermann, Spline approximation methods for the biharmonic Dirichlet problem on non-smooth domains, Operator Theory: Advances and Applications 135, p. 145160, Birkhäuser, 2002. MR1935762 (2003j:65122)

[10] V.D. Didenko, G.L. Pel'ts, On the stability of spline-qualocation method for singular integral equations with conjugation, Differential Equations, v. 29 (1993), p. 1383-1397. MR 1278829 (95c:65222)

[11] R.V. Duduchava, On general singular integral operators of the plane theory of elasticity, Rend. Politec. Torino, 42 (1984) p. 15-41. MR834780 (87f:45016)

[12] R.V. Duduchava, On general singular integral operators of the plane theory of elasticity, Trudy Tbilissk. Mathem. Inst. 82 (1986), p. 45-89 (in Russian) MR884698 (88h:45004)

[13] I. Gohberg, N. Feldman, Convolution Equations and Projection Methods for their Solutions, Akademie Verlag, Berlin, 1974.

[14] I. Gohberg, N. Krupnik, Introduction to the theory of one-dimensional singular integral operators, Birkhäuser, Basel, Boston, Berlin, 1995.

[15] R. Kress, Linear Integral Equations, Springer-Verlag, Berlin, 1999. MR1723850 (2000h:45001)

[16] J. K. Lu, Complex Variable Methods in Plane Elasticity, Series in Pure and Applied Mathematics, v. 22, Singapore, World Scientific, 1995. MR1370444 (96k:73094)

[17] N.I. Muskhelishvili, Fundamental Problems in Theory of Elasticity, Nauka, Moscow, 1966 (in Russian).

[18] N.I. Muskhelishvili, Singular Integral Equations, Nauka, Moscow, 1968 (in Russian). MR0355495 (50:7969)

[19] V.Z. Parton, P.I. Perlin, Integral Equations of Elasticity Theory, Nauka, Moscow, 1977 (in Russian); Integral Equations in Elasticity, Moscow, Mir Publisher, 1982. MR509209 (80a:73001)

[20] P.I. Perlin and Yu.N. Shalyukhin, On the numerical solution of the integral equations of plane elasticity theory, Izv. Akad. Nauk Kasah. SSR, Ser fiz.-mat. 1 (1976) p. 86-88 (in Russian). MR0455783 (56:14017)

[21] P.I. Perlin and Yu.N. Shalyukhin, On the numerical solution of some plane problems in elasticity theory, Prikl. Mech. 15 (1977) p. 83-86 (in Russian).

[22] S. Prössdorf, B. Silbermann, Numerical Analysis for Integral and related Operator Equations, Birkhäuser, Basel, 1991. MR1193030 (94f:65126b)

[23] L.L. Schumaker, Spline functions: basic theory, Krieger publishing, Malabar, Florida, 1993. MR.1226234 (94d:41001)

[24] I.H. Sloan, A quadrature based approach to improving the collocation method, Numer. Math. 54, (1988), p. 41-56. MR960849 (89k:65165)

Mathematics Department, University of Brunei Darussalam, Tungku Be 1410, Brunei

Dipartimento di Matematica, Universitá di Torino, via Carlo Alberto 10, 10123 TORINO, ITALY 